\title{
INEQUALITIES FOR HARMONIC POLYNOMIALS IN TWO AND THREE DIMENSIONS
}

\author{
BY \\ A. C. SCHAEFFER AND G. SZEGÖ
}

In what follows we discuss certain inequalities involving harmonic polynomials of two and of three variables, that is, polynomials $u(x, y)$ and $u(x, y, z)$ which satisfy Laplace's equation $u_{x x}+u_{y y}=0$ and $u_{x x}+u_{y y}+u_{z z}=0$, respectively. The inequalities in question furnish bounds for these polynomials and for their derivatives under proper conditions. Of particular interest are inequalities of the type of S. Bernstein's theorem, as discussed by the second author in a recent paper [7]( $\left.{ }^{1}\right)$.

The first part of the present paper deals with the two-dimensional and the second part with the three-dimensional case. Of fundamental importance throughout the paper is an interpolation formula which is stated and proved in $\$ 2$ of Part I. In fact the results of Part I may be regarded as systematic applications of this formula. Several inequalities of this part are generalizations and refinements of earlier theorems. Most of the problems of the second part are new.

In an Appendix we consider a generalization of the main problem treated in Part II, and another problem which deals with ellipses and is only in loose relationship with the other topics considered in the present paper.

Part I. TWo-dimensional harmonic POLYNomials

\section{Definitions AND Notations}

1. We consider polynomials $u(x, y)$ of degree $n$ in the cartesian coordinates $x, y$ satisfying Laplace's differential equation. Let $n \geqq 1$. Introducing polar coordinates $r, \phi$ we write $u(x, y)=U(r, \phi)$. The standard representation

$$
U(r, \phi)=a_{0}+2 \sum_{m=1}^{n} r^{m}\left(a_{m} \cos m \phi+b_{m} \sin m \phi\right)
$$

is well known. For the sake of simplicity we assume (except in the Appendix, $\S 2$ ) that the $a_{m}$ and $b_{m}$ are real, although many of the following results can be extended to harmonic polynomials with complex coefficients. The boundary function $U(1, \phi)=U(\phi)$ is the most general real trigonometric polynomial of degree $n$,

$$
U(\phi)=a_{0}+2 \sum_{m=1}^{n}\left(a_{m} \cos m \phi+b_{m} \sin m \phi\right) .
$$

Presented to the Society, April 6, 1940; received by the editors September 11, 1940.

(1) Numbers in brackets refer to the Bibliography at the end of the text. 
The conjugate harmonic polynomial $V(r, \phi)$ is unique except for an additive constant; we write

$$
V(r, \phi)=2 \sum_{m=1}^{n} r^{m}\left(-b_{m} \cos m \phi+a_{m} \sin m \phi\right) .
$$

Then $U(r, \phi)$ and $V(r, \phi)$ are the real and imaginary parts, respectively, of the polynomial

$$
F(z)=a_{0}+2 \sum_{m=1}^{n}\left(a_{m}-i b_{m}\right) z^{m}
$$

when $z=r e^{i \phi}$.

2. Let $K_{n}$ be the class of trigonometric polynomials $U(\phi)$ of degree $n$ satisfying the condition.

$$
|U(\nu \pi / n)| \leqq 1, \quad 0 \leqq \nu \leqq 2 n-1 .
$$

Replacing this condition by

$$
U(\nu \pi / n) \geqq 0, \quad 0 \leqq \nu \leqq 2 n-1,
$$

we refer to the corresponding trigonometric polynomials of degree $n$ as those of the class $L_{n}$.

3. Let $\lambda_{0}, \mu_{0}, \lambda_{1}, \mu_{1}, \cdots, \lambda_{n}, \mu_{n}$ be given real numbers with $\mu_{0}=\mu_{n}=0$. In the following development we are interested in obtaining inequalities for the expression

$$
G=\lambda_{0} a_{0}+2 \sum_{m=1}^{n}\left(\lambda_{m} a_{m}+\mu_{m} b_{m}\right)
$$

where $a_{m}$ and $b_{m}$ are the coefficients of an arbitrary trigonometric polynomial of the class $K_{n}$ or $L_{n}$. In these investigations the trigonometric polynomial

$$
\begin{aligned}
g(\phi) & =2 \sum_{m=0}^{n} \prime\left(\lambda_{n-m} \cos m \phi-\mu_{n-m} \sin m \phi\right) \\
& =2 \sum_{m=0}^{n}\left(\lambda_{m} \cos (n-m) \phi-\mu_{m} \sin (n-m) \phi\right)
\end{aligned}
$$

will be of primary importance. Here the symbol $\sum_{m=0}^{\prime n}$ means that the first and last terms $(m=0$ and $m=n)$ are to be multiplied by $\frac{1}{2}$. The above definitions and notations are used throughout the text. Of course (4) can be written in the form

$$
G=\frac{1}{2 \pi} \int_{-\pi}^{\pi} U(\phi)\left\{\lambda_{0}+2 \sum_{m=1}^{n}\left(\lambda_{m} \cos m \phi+\mu_{m} \sin m \phi\right)\right\} d \phi .
$$




\section{FUNDAMENTAL FORMULAS AND THEOREMS}

1. Our starting point is the following important

INTERPOLATION FORMULA. Given the real numbers $\lambda_{0}, \mu_{0}, \lambda_{1}, \mu_{1}, \cdots, \lambda_{n}, \mu_{n}$ with $\mu_{0}=\mu_{n}=0$ and the trigonometric polynomial $U(\phi)$ of degree $n$, we have by using the notations $\left(1^{\prime}\right),(4),(5)$

$$
G=\frac{1}{2 n} \sum_{\nu=0}^{2 n-1}(-1)^{\nu} g(\nu \pi / n) U(\nu \pi / n) .
$$

Special cases of (6) due to M. Riesz [2] and Szegö were discussed in [4]. These cases were used in the proof of S. Bernstein's theorem and the "gradient theorem" (cf. §4) of Szegö, respectively. The interpolation formula of M. Riesz [2] is the special case corresponding to $\lambda_{m}=\cos m \alpha, 0 \leqq m \leqq n$, $\mu_{m}=\sin m \alpha, 0 \leqq m \leqq n-1$; it is the following:

$$
U(\alpha)=2 b_{n} \sin n \alpha
$$

$$
\begin{aligned}
& +\frac{1}{2 n} \sum_{\nu=0}^{2 n-1} U(\nu \pi / n)\{1+2 \cos (\alpha-\nu \pi / n)+2 \cos 2(\alpha-\nu \pi / n)+\cdots \\
& +2 \cos (n-1)(\alpha-\nu \pi / n)+\cos n(\alpha-\nu \pi / n)\} \\
& =2 b_{n} \sin n \alpha+\frac{\sin n \alpha}{2 n} \sum_{\nu=0}^{2 n-1}(-1)^{\nu} U(\nu \pi / n) \cot (\alpha / 2-\nu \pi /(2 n)) .
\end{aligned}
$$

We also note the formula for the corresponding harmonic polynomials (regarding (8) see $[5$, p. 75$])$ :

$$
\begin{aligned}
U(r, \alpha)= & 2 b_{n} r^{n} \sin n \alpha+\frac{1}{2 n} \sum_{\nu=0}^{2 n-1} U(\nu \pi / n)\{1+2 r \cos (\alpha-\nu \pi / n) \\
& +2 r^{2} \cos 2(\alpha-\nu \pi / n)+\cdots \\
& \left.+2 r^{n-1} \cos (n-1)(\alpha-\nu \pi / n)+r^{n} \cos n(\alpha-\nu \pi / n)\right\} \\
V(r, \alpha)= & -2 b_{n} r^{n} \cos n \alpha+\frac{1}{2 n} \sum_{\nu=0}^{2 n-1} U(\nu \pi / n)\{2 r \sin (\alpha-\nu \pi / n) \\
& +2 r^{2} \sin 2(\alpha-\nu \pi / n)+\cdots \\
& \left.+2 r^{n-1} \sin (n-1)(\alpha-\nu \pi / n)+r^{n} \sin n(\alpha-\nu \pi / n)\right\}
\end{aligned}
$$

2. From the formula of M. Riesz, (6) can easily be derived. A direct proof of (6) can be based on the well known remark that for any trigonometric polynomial $f(\phi)$ of degree $2 n-1$,

$$
\frac{1}{2 n} \sum_{\nu=0}^{2 n-1} f(\nu \pi / n)=\frac{1}{2 \pi} \int_{-\pi}^{\pi} f(\phi) d \phi .
$$

Now, 


$$
\begin{aligned}
& (-1)^{\nu} \cos [(n-m) \nu \pi / n]=\cos (m \nu \pi / n), \\
& (-1)^{\nu} \sin [(n-m) \nu \pi / n]=-\sin (m \nu \pi / n),
\end{aligned}
$$

so making use of (5), the right-hand side of (6) is equal to

$$
\frac{1}{2 n} \sum_{\nu=0}^{2 n-1} \sum_{m=0}^{n} 2 U(\nu \pi / n)\left(\lambda_{m} \cos (m \nu \pi / n)+\mu_{m} \sin (m \nu \pi / n)\right) .
$$

On changing the order of summation and using (10) we find that this is equal to $G$. It has to be taken into account that

$$
\frac{1}{2 n} \sum_{\nu=0}^{2 n-1} \lambda_{n} \cos (n \nu \pi / n)\left(2 a_{n} \cos (n \nu \pi / n)+2 b_{n} \sin (n \nu \pi / n)\right)=2 \lambda_{n} a_{n} .
$$

3. As a first consequence of this interpolation formula we prove

TheOREM A. Let $\lambda_{0}, \mu_{0}, \lambda_{1}, \mu_{1}, \cdots, \lambda_{n}, \mu_{n}$ be given real numbers, $\mu_{0}=\mu_{n}=0$, for which

$$
g(\nu \pi / n) \geqq 0, \quad 0 \leqq \nu \leqq 2 n-1 .
$$

If $U(\phi)$ belongs to the class $K_{n}$, then

$$
|G|=\left|\lambda_{0} a_{0}+2 \sum_{m=1}^{n}\left(a_{m} \lambda_{m}+\mu_{m} b_{m}\right)\right| \leqq \lambda_{n} .
$$

If (12) is not satisfied (that is, if for at least one value of $\nu$ the sign $<$ holds), then there is a member of $K_{n}$ for which $G>\lambda_{n}$.

REMARK. Let $\nu_{1}, \nu_{2}, \cdots, \nu_{l}$ denote all the values of $\nu, 0 \leqq \nu \leqq 2 n-1$, for which in (12) the sign > holds. Then $|G|=\lambda_{n}$ if and only if $(-1)^{\nu} U(\nu \pi / n)=\gamma$, $\nu=\nu_{1}, \nu_{2}, \cdots, \nu_{l}$. Here $\gamma$ represents either +1 or -1 . If in all the inequalities (12) the sign > holds, then $|G|=\lambda_{n}$ if and only if $U(\phi)= \pm \cos n \phi+c \sin n \phi$, c real.

This result was proved by Szegö in the less precise form that (13) follows if, in place of (12) and the condition that $U(\phi)$ belongs to $K_{n}$, we have $g(\phi) \geqq 0$ and $|U(\phi)| \leqq 1$ for all real $\phi$. The above generalization of the theorem was proved by S. Bernstein [1] using the method of best approximation. The present method is similar to Szegö's.

4. Inequality (13) is a simple consequence of the fundamental interpolation formula; for under the hypotheses of Theorem A,

$$
|G|=\left|\frac{1}{2 n} \sum_{\nu=0}^{2 n-1}(-1)^{\nu} g(\nu \pi / n) U(\nu \pi / n)\right| \leqq \frac{1}{2 n} \sum_{\nu=0}^{2 n-1} g(\nu \pi / n)=\lambda_{n} .
$$

Now let $g(k \pi / n)<0$ for a special value of $k$. We construct a trigonometric polynomial of class $K_{n}$ for which $G>\lambda_{n}$. The function $f(\phi)$ 
$=(2 n)^{-1} \sin n \phi \cot (\phi / 2)$ is a trigonometric polynomial of degree $n$ vanishing at $\phi=\nu \pi / n, 1 \leqq \nu \leqq 2 n-1$, and equal to 1 at $\phi=0$. [This function serves as a basis for the interpolation formula of M. Riesz. See (7).] Then the trigonometric polynomial defined by

$$
U(\phi)=\sum_{\nu=0}^{2 n-1}(-1)^{\nu} f(\phi-\nu \pi / n) \operatorname{sgn} g(\nu \pi / n)
$$

belongs to $K_{n}$. But from (6)

$$
G=\frac{1}{2 n} \sum_{\nu=0}^{2 n-1}|g(\nu \pi / n)|>\frac{1}{2 n} \sum_{\nu=0}^{2 n-1} g(\nu \pi / n)=\lambda_{n} .
$$

But more than this is true. If $g(k \pi / n)<0$ for a special $k$, there is a trigonometric polynomial of degree $n$ which is bounded by 1 for all real $\phi$, but for which $G>\lambda_{n}$. To show this let $\epsilon$ be a small positive number and let

$$
U_{\epsilon}(\phi)=\left(1-\epsilon-\epsilon^{3 / 2}\right)(\cos n \phi+\epsilon U(\phi))
$$

where $U(\phi)$ is defined by (15). Suppose $U_{\epsilon}(\phi)$ attains its maximum at $\phi_{1}$, which of course must lie within a distance $\pi /(2 n)$ of some point $\nu \pi / n$. Then

$$
\epsilon U^{\prime}\left(\phi_{1}\right)=n \sin n \phi_{1},
$$

so, from the inequality $(\sin \theta) / \theta \geqq 2 / \pi,|\theta| \leqq \pi / 2$,

$$
\epsilon\left|U^{\prime}\left(\phi_{1}\right)\right|=n\left|\sin n\left(\phi_{1}-\nu \pi / n\right)\right| \geqq(2 n / \pi)\left|\phi_{1}-\nu \pi / n\right| \text {. }
$$

Thus $\phi_{1}$ lies in fact within a distance $c_{1} \epsilon$ of some point $\nu \pi / n, c_{1}$ independent of $\epsilon$. Then the mean value theorem furnishes

$$
U_{\epsilon}\left(\phi_{1}\right)-U_{\epsilon}(\nu \pi / n)=\left(1-\epsilon-\epsilon^{3 / 2}\right)\left(\phi_{1}-\nu \pi / n\right)\left(-n \sin n \theta+\epsilon U^{\prime}(\theta)\right)
$$

where $|\theta-\nu \pi / n| \leqq\left|\phi_{1}-\nu \pi / n\right| \leqq c_{1} \epsilon$. The last factor on the right will be less than $c_{2} \epsilon, c_{2}$ independent of $\epsilon$, so

$$
\left|U_{\epsilon}\left(\phi_{1}\right)\right|<\left(1-\epsilon-\epsilon^{3 / 2}\right)\left(1+\epsilon+c_{1} c_{2} \epsilon^{2}\right)<1
$$

for all small $\epsilon$.

But

$$
\begin{aligned}
G=G_{\epsilon} & =\left(1-\epsilon-\epsilon^{3 / 2}\right) /(2 n) \sum_{\nu=0}^{2 n-1} g(\nu \pi / n)\{1+\epsilon \operatorname{sgn} g(\nu \pi / n)\} \\
& \geqq\left(1-\epsilon-\epsilon^{3 / 2}\right)\left\{\lambda_{n}+\epsilon \lambda_{n}+(\epsilon / n)|g(k \pi / n)|\right\}>\lambda_{n}
\end{aligned}
$$

if $\epsilon$ is small enough.

If all $g(\nu \pi / n)$ are greater than zero the equality occurs in (14) if and only if $(-1)^{\nu} U(\nu \pi / n)=\gamma, 0 \leqq \nu \leqq 2 n-1$. Here $\gamma$ represents +1 or -1 . In this case, however, the function $\gamma U(\phi)-\cos n \phi$ vanishes at $\nu \pi / n, 0 \leqq \nu \leqq 2 n-1$, and so is identically equal to $c \sin n \phi$ where $c$ is a real constant. The more general statement in the Remark to Theorem A is also clear. The values of $U(\nu \pi / n)$ for the remaining $\nu \neq \nu_{1}, \nu_{2}, \cdots, \nu_{l}$ are obviously immaterial.

5. A result which is closely related to Theorem $A$ is the following:

Theorem B. Let $\lambda_{0}, \mu_{0}, \lambda_{1}, \mu_{1}, \cdots, \lambda_{n}, \mu_{n}$ be given real numbers, $\mu_{0}=\mu_{n}=0$, for which 


$$
(-1)^{\nu} g(\nu \pi / n) \geqq 0,
$$$$
0 \leqq \nu \leqq 2 n-1 \text {. }
$$

If $U(\phi)$ is a member of the class $L_{n}$, then

$$
G=\lambda_{0} a_{0}+2 \sum_{m=1}^{n}\left(\lambda_{m} a_{m}+\mu_{m} b_{m}\right) \geqq 0 .
$$

If (17) is not satisfied (that is, if for at least one value of $\nu$ the sign $<$ holds), then there is a member of $L_{n}$ for which $G<0$.

REMARK. Let $\nu_{1}, \nu_{2}, \cdots, \nu_{l}$ denote all the values of $\nu, 0 \leqq \nu \leqq 2 n-1$, for which in (17) the sign $>$ holds. Then $G=0$ if and only if $U(\nu \pi / n)=0, \nu=\nu_{1}, \nu_{2}, \cdots, \nu_{l}$. If in all the inequalities (17) the sign $>$ holds, then $G=0$ if and only if

$$
U(\phi) \equiv c \sin n \phi,
$$

c real.

Inequality (18) follows immediately from (6). Now suppose $(-1)^{k} g(k \pi / n)$ $<0$ for a special value of $k$. Then

$$
U(\phi)=(1 / 2 n) \sin n(\phi-k \pi / n) \cot \frac{1}{2}(\phi-k \pi / n)
$$

belongs to the class $L_{n}$, and $U(k \pi / n)=1$. But for this function

$$
G=\frac{1}{2 n} \sum_{\nu=0}^{2 n-1}(-1)^{\nu} g(\nu \pi / n) U(\nu \pi / n)=\frac{1}{2 n}(-1)^{k} g(k \pi / n) U(k \pi / n)<0 .
$$

If for all $\nu,(-1)^{\nu} g(\nu \pi / n)>0$, the equality occurs in (18) if and only if $U(\nu \pi / n)=0,0 \leqq \nu \leqq 2 n-1$. But this implies that $U(\phi) \equiv c \sin n \phi, c$ real. The more general assertion of the Remark to Theorem B is also clear.

6. Theorems A and B are "dual" theorems in the following sense. Let $\lambda_{0}, \mu_{0}, \lambda_{1}, \mu_{1}, \cdots, \lambda_{n}, \mu_{n} ; \mu_{0}=\mu_{n}=0$; be given real numbers, and suppose that the function $g_{1}(\phi)=2 \sum_{m=0}^{\prime n}\left(\lambda_{n-m} \cos m \phi-\mu_{n-m} \sin m \phi\right)$ satisfies (12). If $\left\{\lambda_{m}{ }^{\prime}, \mu_{m}{ }^{\prime}\right\}$ is a new sequence defined by $\lambda_{m}^{\prime}=\lambda_{n-m}, \mu_{m}^{\prime}=-\mu_{n-m}$, the function $g_{2}(\phi)=2 \sum_{m=0}^{\prime n}\left(\lambda_{n-m}^{\prime} \cos m \phi-\mu_{n-m}^{\prime} \sin m \phi\right)$ will satisfy (17). In fact it follows from (11) that $g_{2}(\nu \pi / n)=(-1)^{\nu} g_{1}(\nu \pi / n)$. Thus if the sequence $\left\{\lambda_{m}, \mu_{m}\right\}$ is such that Theorem $\mathrm{A}$ is applicable to polynomials of the class $K_{n}$, the sequence $\left\{\lambda_{m}^{\prime}, \mu_{m}^{\prime}\right\}$ will be such that Theorem B will be applicable to polynomials of the class $L_{n}$. The converse is also true.

7. Finally we note some identities which will be useful in the later considerations (cf. [5, p. 75]):

$$
\begin{aligned}
A & =2 \sum_{m=0}^{n} r^{m} \cos m r \\
& =\frac{\left(1-r^{2}\right)\left(1-r^{n} \cos n \phi\right)+2 r^{n+1} \sin n \phi \sin \phi}{1-2 r \cos \phi+r^{2}},
\end{aligned}
$$




$$
\begin{aligned}
B & =2 \sum_{m=0}^{n} r^{n-m} \cos m \phi \\
& =\frac{\left(r^{2}-1\right)\left(r^{n}-\cos n \phi\right)+2 r \sin n \phi \sin \phi}{1-2 r \cos \phi+r^{2}} \\
C & =2 \sum_{m=0}^{n}{ }^{\prime} \cos m \phi=\sin n \phi \cot (\phi / 2)
\end{aligned}
$$

For $\phi=\nu \pi / n, \nu$ an integer, we have

$$
\begin{array}{llll}
\operatorname{sgn} A=+1, & \operatorname{sgn} B=(-1)^{\nu} & \text { if } & r<1, \\
\operatorname{sgn} A=(-1)^{\nu}, & \operatorname{sgn} B=+1 & \text { if } & r>1,
\end{array}
$$

and

$$
\operatorname{sgn} C=0 \text { or }+1
$$

according as $\nu$ is not or is divisible by $2 n$.

\section{Applications}

1. In this paragraph we consider several applications of Theorems A and B.

I. Let $U(r, \phi)$ be a harmonic polynomial and let $U(1, \phi)=U(\phi)$ belong to the class $K_{n}$. Then for $R>1$

$$
\left|U(R, \nu \pi / n)+\delta R^{n} U\left(R^{-1}, \nu \pi / n\right)\right| \leqq R^{n}+\delta, \quad 0 \leqq \nu \leqq 2 n-1,
$$

where $\delta=+1$ or -1 . In the special case $\nu=\nu_{0}$ of (25) the equality occurs if and only if $U(1, \nu \pi / n)=\gamma$ either for all even $\nu$ or for all odd $\nu$ according as $\nu_{0}+\frac{1}{2}(1-\delta)$ is even or odd; here $\gamma=+1$ or -1 .

From this follows

$\mathrm{I}^{\prime}$. Let $U(r, \phi)$ be a harmonic polynomial of degree $n$ satisfying the condition $|U(1, \phi)| \leqq 1, \phi$ arbitrary real. Then for $R>1$,

$$
\left|U(R, \phi)+\delta R^{n} U\left(R^{-1}, \phi\right)\right| \leqq R^{n}+\delta, \quad \phi \text { arbitrary real, }
$$

where $\delta=+1$ or -1 . The equality occurs in $\left(25^{\prime}\right)$ if and only if $\pm U(r, \phi)$ $\equiv 1-\lambda+\lambda r^{n} \cos n\left(\phi-\phi_{0}\right)$ where $\phi_{0}$ is real, $0 \leqq \lambda \leqq 1$.

To prove I it will be sufficient to consider the special case $\nu=0$. Let

$$
\lambda_{m}=R^{m}+\delta R^{n-m}, \quad \mu_{m}=0, \quad 0 \leqq m \leqq n,
$$

where $\delta= \pm 1$. Then

$$
g(\phi)=2 \sum_{m=0}^{n} R^{n-m} \cos m \phi+2 \delta \sum_{m=0}^{n} R^{n-m} \cos (n-m) \phi,
$$


and making use of (11) we obtain

$$
g(\nu \pi / n)=2\left(1+\delta(-1)^{\nu}\right) \sum_{m=0}^{n}{ }^{\prime} R^{n-m} \cos (m \nu \pi / n) .
$$

The last sum is positive if $R>1$ [see (23)]. Consequently $g(\nu \pi / n)$ is greater than zero either for all even $\nu$ or for all odd $\nu$, and vanishes for the remaining $\nu$. Then (13) furnishes

$$
\left|a_{0}\left(1+\delta R^{n}\right)+2 \sum_{m=1}^{n} a_{m}\left(R^{m}+\delta R^{n-m}\right)\right| \leqq R^{n}+\delta
$$

that is, inequality (25). The conditions of equality in (25) follow from the Remark to Theorem A. Suppose for example that $\nu_{0}=0$ and $\delta=+1$. Then (26) shows that $g(\nu \pi / n)>0$ for even $\nu$ and $=0$ for odd $\nu$. Thus the equality holds if and only if $U(1, \nu \pi / n)=\gamma$ for even $\nu$, whereas $U(1, \nu \pi / n)$ is arbitrary for odd $\nu$.

2. Inequality (25) is true if $\delta$ is not required to be \pm 1 , but is allowed to have any value between -1 and +1 . This follows by the argument used in the proof of $I$, since (26) is non-negative if $-1 \leqq \delta \leqq 1$. It is interesting to note, however, that if $-1<\delta<1$ inequality (25) may become an equality, but under conditions different from those stated in I. For if $-1<\delta<1$, then $g(\nu \pi / n)>0$ for all $\nu$, so (25) becomes an equality for the narrower class of functions $U(r, \phi)= \pm r^{n} \cos n \phi+c r^{n} \sin n \phi, c$ real.

3. Inequality $\left(25^{\prime}\right)$ is obtained from (25) in a familiar way. However the question of $\left(25^{\prime}\right)$ becoming an equality requires special consideration. Let the sign " $="$ hold in $\left(25^{\prime}\right)$ for $\phi=0$, and let $\delta=+1$. In virtue of the result on the conditions of equality in I we have $U(1, \nu \pi / n)=\gamma$ for $\nu=0,2,4, \cdots, 2 n-2$. Then the derivative of $U(1, \phi)$ with respect to $\phi$ must vanish at the same points. Thus, if $\lambda$ is any real constant the function $f(\phi)=\gamma U(1, \phi)-\cos n \phi$ $-\lambda(1-\cos n \phi)$ will have $n$ double zeros, $\bmod 2 \pi$. But choosing $\lambda=\gamma U(1, \pi /(2 n))$ there will also be a zero at $\phi=\pi / 2 n$; that is, $f(\phi)$ will have $2 n+1 \operatorname{zeros}, \bmod 2 \pi$, and so will vanish identically. This furnishes $\gamma U(1, \phi) \equiv \lambda+(1-\lambda) \cos n \phi$; in order that $|U(1, \phi)| \leqq 1$ be satisfied it is necessary that $0 \leqq \lambda \leqq 1$; finally we substitute $1-\lambda$ for $\lambda$.

4. The dual of $I$ is

II. Let $U(r, \phi)$ be a harmonic polynomial and let $U(1, \phi)=U(\phi)$ belong to the class $L_{n}$. Then for $R>1$

$$
|U(R, \nu \pi / n)| \leqq R^{n} U\left(R^{-1}, \nu \pi / n\right), \quad 0 \leqq \nu \leqq 2 n-1 .
$$

The equality holds if and only if $U(1, \nu \pi / n)=0$ either for all even $\nu$ or for all odd $\nu$.

A consequence of this is the following theorem of Szegö [3, p. 333]: 
II'. Let $U(r, \phi)$ be a harmonic polynomial of degree $n$ satisfying $U(1, \phi) \geqq 0$, $\phi$ arbitrary real. Then for $R>1$,

$$
|U(R, \phi)| \leqq R^{n} U\left(R^{-1}, \phi\right), \quad \phi \text { arbitrary real. }
$$

The equality occurs if and only if $U(r, \phi) \equiv \lambda\left\{1+r^{n} \cos n\left(\phi-\phi_{0}\right)\right\}, \phi_{0}$ and $\lambda$ real, $\lambda \geqq 0$.

To prove II it is again sufficient to consider the case $\nu=0$. Let $\lambda_{m}=R^{n-m}$ $+\delta R^{m}, \mu_{m}=0$ where, as before, $R>1$ and $\delta= \pm 1$. Then by the remark of $\$ 2.6$ and by (26), the function $g(\phi)$ constructed with the present choice of $\lambda_{m}$ and $\mu_{m}$ will satisfy (17). More precisely, $(-1)^{\nu} g(\nu \pi / n)$ will be greater than zero either for all even $\nu$ or for all odd $\nu$, and will vanish for the remaining $\nu$ in the range $0 \leqq \nu \leqq 2 n-1$. Thus, according to Theorem $\mathrm{B}$

or

$$
a_{0}\left(R^{n}+\delta\right)+2 \sum_{m=1}^{n}\left(R^{n-m}+\delta R^{m}\right) a_{m} \geqq 0
$$

$$
R^{n} U\left(R^{-1}, 0\right)+\delta U(R, 0) \geqq 0 .
$$

This shows that (27) is true for $\nu=0$.

If (27) is an equality (for $\nu=0$ ) then (28) will be also for proper choice of $\delta$. Then the Remark to Theorem B shows that $U(1, \nu \pi / n)$ must vanish, either for all even $\nu$ or for all odd $\nu$. Conversely, if $U(1, \nu \pi / n)=0, \nu$ even, we choose $\delta=+1$; then according to (26), $g(\nu \pi / n)=0$ for all odd $\nu$ so that in (28) the equality holds. The same is true in (27). The argument is similar if $U(1, \nu \pi / n)=0$ for all odd $\nu$.

5. From the extensions of $\mathrm{I}$ and $\mathrm{I}^{\prime}$ given in $\$ 3.2$ we obtain for $\delta=0$ :

If $U(r, \phi)$ satisfies the conditions of $\mathrm{I}$, then for $R>1$

$$
|U(R, \nu \pi / n)| \leqq R^{n}, \quad 0 \leqq \nu \leqq 2 n-1 .
$$

If $U(R, \phi)$ satisfies the conditions of $\mathrm{I}^{\prime}$, then for $R>1$

$$
|U(R, \phi)| \leqq R^{n}, \quad \quad \phi \text { arbitrary real. }
$$

The second of these is already known [3]. In the second part of the present paper we deal with the space analogues of (29) and (30).

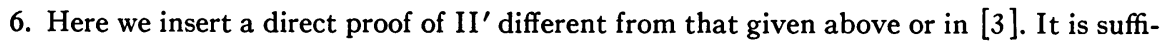
cient to prove $\left(27^{\prime}\right)$ for the special case $\phi=0$; also considering $\frac{1}{2}\{U(r, \phi)+U(r,-\phi)\}$ instead of $U(r, \phi)$ it suffices to prove $\left(27^{\prime}\right)$ for the case in which $\phi=0$ and $U(r, \phi)$ contains only cosine terms, that is, for

$$
U(r, \phi)=a_{0}+2 \sum_{m=1}^{n} a_{m} r^{m} \cos m \phi .
$$

Finally we can assume $U(r, \phi)>0$ for $0 \leqq r<1$. Now $U(r, \phi)$ is the real part of the polynomial

$$
F(z)=F\left(r e^{i \phi}\right)=a_{0}+2 \sum_{m=0}^{n} a_{m} z^{m} \neq 0, \quad|z|<1,
$$


so $p(z)=z^{n} F\left(z^{-1}\right) / F(z)$ is regular for $|z|<1$. According to a familiar argument, $p(z)$ has the constant modulus 1 for $|z|=1$, so

$$
\left|z^{n} F\left(z^{-1}\right)\right|<F(z), \quad|z|<1,
$$

unless $p(z) \equiv$ const. $= \pm 1$. This inequality furnishes $\left(27^{\prime}\right)$ by choosing $z=R^{-1}, R>1$. The assumption $p(z) \equiv \pm 1$ implies $a_{0}= \pm 2 a_{n}$, from which, since $U(1, \phi) \geqq 0$, we conclude $\left({ }^{2}\right)$ that $U(1, \phi) \equiv \lambda(1 \pm \cos n \phi), \lambda>0$.

Returning again to the general case, the equality can hold in $\left(27^{\prime}\right)$ at $\phi=0$ only if

$$
U(1, \phi) \equiv \lambda(1 \pm \cos n \phi)+S(\phi)
$$

where $S(\phi)$ is a sine polynomial. This again implies $U(1, \phi) \equiv \lambda(1 \pm \cos n \phi)$.

7. An inequality of $S$. Bernstein states:

Let $U(\phi)$ be a trigonometric polynomial of degree $n$ satisfying $|U(1, \phi)| \leqq 1$, $\phi$ arbitrary real. Then $\left|U^{\prime}(\phi)\right| \leqq n, \phi$ arbitrary real, with the equality if and only if $U(\phi) \equiv \cos n\left(\phi-\phi_{0}\right), \phi_{0}$ real.

This will be a consequence of the following $\left({ }^{3}\right)$ :

Let $U(\phi)$ belong to the class $K_{n}$. Then $\left|U^{\prime}(\pi /(2 n))\right| \leqq n$ with the equality if and only if $U(\phi) \equiv \pm \cos n \phi+c \sin n \phi, c$ real.

To prove this, let

$$
\lambda_{m}=m \sin (m \pi /(2 n)), \quad \mu_{m}=-m \cos (m \pi /(2 n)), \quad 0 \leqq m \leqq n .
$$

Then

$$
\begin{aligned}
g(\phi) & =2 \sum_{m=0}^{n}(n-m)\{\cos (m \pi /(2 n)) \cos m \phi+\sin (m \pi /(2 n)) \sin m \phi\} \\
& =\left\{\frac{\sin \frac{1}{2} n(\pi /(2 n)-\phi)}{\sin \frac{1}{2}(\pi /(2 n)-\phi)}\right\}^{2}
\end{aligned}
$$

is the classical Fejér kernel, and $g(\nu \pi / n)>0$. It follows from Theorem A that

$$
\left|2 \sum_{m=1}^{n} m\left\{a_{m} \sin (m \pi /(2 n))-b_{m} \cos (m \pi /(2 n))\right\}\right| \leqq \lambda_{n}
$$

which is the assertion.

\section{THE "GRADIENT THEOREM"}

1. Szegö proved the following results [4] which for ease of reference we call the "gradient theorem" and the "generalized gradient theorem." They are refinements of S. Bernstein's theorem.

(2) L. Fejér, Über trigonometrische Polynome, Journal für die reine und angewandte Mathematik, vol. 146 (1915), pp. 53-82; p. 72.

(3) Cf. G. P6lya and G. Szegö, Aufgaben und Lehrsätze aus der Analysis, vol. 2, 1925, Problem IV, p. 201, and pp. 35, 218, 219; see in particular p. 219. 
GRADIENT THEOREM. If $U(r, \phi)$ is a harmonic polynomial of degree $n$ satisfying $|U(r, \phi)| \leqq 1$ in $r \leqq 1$, then $|\operatorname{grad} U| \leqq n$ in $r \leqq 1$. The equality holds if and only if $U(r, \phi) \equiv r^{n} \cos n\left(\phi-\phi_{0}\right), \phi_{0}$ real.

GENERALIZED GRADIENT THEOREM. If $U(r, \phi)$ is a harmonic polynomial of degree $n$ satisfying $|U(r, \phi)| \leqq 1$ in $r \leqq 1$, then

$$
|\operatorname{grad} U(r, \phi)|+n\left|\sigma_{n}(r, \phi)\right| \leqq n \quad \text { in } r \leqq 1 .
$$

Except at points $(1, \phi)$ where $|U(1, \phi)|=1$ the equality occurs if and only if $\pm U(r, \phi) \equiv 1-\lambda+\lambda r^{n} \cos n\left(\phi-\phi_{0}\right), \lambda$ and $\phi_{0}$ real, $0 \leqq \lambda \leqq 1$.

Here and henceforth $\sigma_{n}(r, \phi)$ denotes the $n$th Cesàro means of first order; if $U(r, \phi)$ is given by (1), then

$$
\sigma_{n}(r, \phi)=a_{0}+2 \sum_{m=1}^{n}(1-m / n) r^{m}\left(a_{m} \cos m \phi+b_{m} \sin m \phi\right) .
$$

If $U(r, \phi)$ and $V(r, \phi)$ are conjugate harmonic polynomials we note that

$$
\begin{aligned}
|\operatorname{grad} U| & =\left\{\left(r^{-1} U_{\phi}\right)^{2}+\left(r^{-1} V_{\phi}\right)^{2}\right\}^{1 / 2}=\left\{\left(U_{r}\right)^{2}+\left(r^{-1} U_{\phi}\right)^{2}\right\}^{1 / 2} \\
& =\left\{\left(U_{r}\right)^{2}+\left(V_{r}\right)^{2}\right\}^{1 / 2} .
\end{aligned}
$$

In particular it follows from the gradient theorem that $\left|U_{r}\right| \leqq n$ and $\left|U_{\phi}\right| \leqq n$. The last inequality is S. Bernstein's theorem.

2. Incidentally the conditions of equality for the generalized gradient theorem were not discussed in [4]. To obtain this theorem by the method of the present paper we first show that

$$
\left|\cos \alpha U_{r}(1, \alpha / n)+\sin \alpha V_{r}(1, \alpha / n)+n \sigma_{n}(1, \alpha / n)\right| \leqq n
$$

for every real $\alpha$. Now $U(r, \phi-\beta), \beta$ real, also satisfies the conditions of the generalized gradient theorem; so (34) will imply that

$$
\left|\cos \alpha U_{r}(1, \phi)+\sin \alpha V_{r}(1, \phi)+n \sigma_{n}(1, \phi)\right| \leqq n .
$$

Inequality (31) will be an immediate consequence of this, at least for $r=1$.

To prove (34) let

$$
\begin{aligned}
& \lambda_{m}=m \cos (\alpha-m \alpha / n)+(n-m) \cos (m \alpha / n), \\
& \mu_{m}=-m \sin (\alpha-m \alpha / n)+(n-m) \sin (m \alpha / n) .
\end{aligned}
$$

Then

$$
g(\phi)=2 \sum_{m=0}^{n}(n-m) \cos (m \phi-m \alpha / n)+2 \sum_{m=0}^{n}(n-m) \cos (m \alpha / n+n \phi-m \phi)
$$




$$
\begin{aligned}
g(\nu \pi / n) & =2\left\{1+(-1)^{\nu}\right\} \sum_{m=0}^{n}(n-m) \cos (m(\alpha-\nu \pi) / n) \\
& =\left\{1+(-1)^{\nu}\right\}\left\{\frac{\sin ((\alpha-\nu \pi) / 2)}{\sin ((\alpha-\nu \pi) /(2 n))}\right\}^{2} \geqq 0 .
\end{aligned}
$$

A little algebraic manipulation will show that $|G|$ is equal to the left-hand side of (34), hence (34) is a consequence of Theorem A.

To discuss the conditions of equality note that $g(\nu \pi / n)=0$ if and only if $\nu$ is odd or $\alpha-\nu \pi$ is of the form $\alpha-\nu \pi=2 k \pi$ where $k$ is an integer not divisible by $n$. Consequently, if $\alpha / \pi$ is not an integer (34) is an equality if and only if $U(1, \nu \pi / n)=\gamma, \gamma= \pm 1$, for all even $\nu$. If $\alpha / \pi$ is an integer the same is true unless $\alpha \equiv 0(\bmod 2 \pi)$. In this exceptional case we see that the left-hand side of $(34)$ is equal to

$$
\left|U_{r}(1, \alpha / n)+n \sigma_{n}(1, \alpha / n)\right|=|n U(1, \alpha / n)|
$$

and this is equal to $n$ only at points where $U(1, \alpha / n)= \pm 1$.

The same argument which was used in $\$ 3.3$ shows that at points on the unit circle where $|U(1, \phi)|<1$ the equality holds only if $\pm U(1, \phi) \equiv 1-\lambda$ $+\lambda \cos n\left(\phi-\phi_{0}\right), 0 \leqq \lambda \leqq 1, \phi_{0}$ real.

This proves the generalized gradient theorem for $r=1$. If $U_{s}$ denotes the directional derivative of $U$ in a fixed direction $s$, then $U_{s}(r, \phi)+n \sigma_{n}(r, \phi)$ is a harmonic function. It follows that

$$
\left|U_{s}(r, \phi)+n \sigma_{n}(r, \phi)\right| \leqq n,
$$

But now, if $(r, \phi)$ is a fixed point in the interior of the unit circle and $s$ is allowed to vary we have

$$
|\operatorname{grad} U(r, \phi)|+n\left|\sigma_{n}(r, \phi)\right|=\max \left|U_{s}(r, \phi)+n \sigma_{n}(r, \phi)\right| \leqq n .
$$

According to the maximum principle, (35) can be an equality only if the corresponding equality is identically satisfied. In this case $U_{s}(1, \phi)+n \sigma_{n}(1, \phi)$ $\equiv \pm n$ for all real $\phi$ so $\pm U(1, \phi) \equiv 1-\lambda+\lambda \cos n\left(\phi-\phi_{0}\right)$.

3 . In the further course of this paragraph we prove the following generalization of the gradient theorems.

III. Let $U(r, \phi)$ be a harmonic polynomial and let $U(1, \phi)=U(\phi)$ belong to the class $K_{n}$. Then if $p>q \geqq 0, p+q \geqq 2$, and $\alpha$ is real,

$$
\begin{aligned}
|\cos \alpha\{U(p, \alpha / n)-U(q, \alpha / n)\}+\sin \alpha\{V(p, \alpha / n)-V(q, \alpha / n)\}| \\
+\left|p^{n} U\left(p^{-1}, \alpha / n\right)-q^{n} U\left(q^{-1}, \alpha / n\right)\right| \leqq p^{n}-q^{n} .
\end{aligned}
$$

The equality occurs if and only if $U(1, \nu \pi / n)=\gamma, \gamma= \pm 1$, either for all even $\nu$ or for all odd $\nu$, the only exception being the case $n=2, p+q=2, \alpha / \pi$ an integer.

From this follows: 
III'. Let $U(r, \phi)$ be a harmonic polynomial of degree $n$ satisfying $|U(1, \phi)|$ $\leqq 1, \phi$ arbitrary real. Then if $p>q \geqq 0, p+q \geqq 2$,

$$
\begin{aligned}
{\left[\{U(p, \phi)-U(q, \phi)\}^{2}+\{V(p, \phi)-V(q, \phi)\}^{2}\right]^{1 / 2} } \\
\quad+\left|p^{n} U\left(p^{-1}, \phi\right)-q^{n} U\left(q^{-1}, \phi\right)\right| \leqq p^{n}-q^{n} .
\end{aligned}
$$

The equality occurs if and only if $\pm U(r, \phi) \equiv \lambda+(1-\lambda) r^{n} \cos n\left(\phi-\phi_{0}\right), \lambda$ and $\phi_{0}$ real, $0 \leqq \lambda \leqq 1$, the only exception being the case $n=2, p+q=2\left({ }^{4}\right)$.

These results include the generalized gradient theorem as a limiting case. For, let each side of (36) and (36') be divided by $p-q$ and let $p \rightarrow 1, q \rightarrow 1$. We obtain

$$
\left|\cos \alpha U_{r}(1, \alpha / n)+\sin \alpha V_{r}(1, \alpha / n)\right|+n\left|\sigma_{n}(1, \alpha / n)\right| \leqq n
$$

and

$$
\left[\left\{U_{r}(1, \phi)\right\}^{2}+\left\{V_{r}(1, \phi)\right\}^{2}\right]^{1 / 2}+n\left|\sigma_{n}(1, \phi)\right| \leqq n,
$$

respectively. The first inequality states slightly more than (34); the second inequality is the generalized gradient theorem.

4. To prove III let

$$
\begin{aligned}
& \lambda_{m}=\left(p^{m}-q^{m}\right) \cos (\alpha-m \alpha / n)+\delta\left(p^{n-m}-q^{n-m}\right) \cos (m \alpha / n), \\
& \mu_{m}=-\left(p^{m}-q^{m}\right) \sin (\alpha-m \alpha / n)+\delta\left(p^{n-m}-q^{n-m}\right) \sin (m \alpha / n) .
\end{aligned}
$$

Here $\delta$ represents +1 or -1 . Then

$$
g(\phi)=\sum_{m=0}^{n}\left(p^{n-m}-q^{n-m}\right)\{\cos (m \alpha / n-m \phi)+\delta \cos (m \alpha / n+n \phi-m \phi)\}
$$

so

$$
g(\nu \pi / n)=\left\{1+(-1)^{\nu} \delta\right\} \sum_{m=0}^{n}\left(p^{n-m}-q^{n-m}\right) \cos (m \alpha / n-m \nu \pi / n) .
$$

If we can show that

$$
\begin{aligned}
F(\phi) & =\sum_{m=0}^{n}\left(p^{n-m}-q^{n-m}\right) \cos m \phi \\
& =\sum_{k=1}^{n}\left[\left\{(p-1)^{k}-(q-1)^{k}\right\} \sum_{m=0}^{n} C_{n-m, k} \cos m \phi\right] \geqq 0, \quad \phi \text { real, }
\end{aligned}
$$

it will follow that $g(\phi)$ satisfies (12). But $\sum_{m=0}^{\prime n} C_{n-m, k} \cos m \phi \geqq 0$ if $k \geqq 1$, this expression being, apart from a positive factor, the Cesàro means of $k$ th order of the series $\frac{1}{2}+\cos \phi+\cos 2 \phi+\cdots$. Moreover, from the hypotheses of III

(4) In this case $\left(36^{\prime}\right)$ is an equality for $\phi=\phi^{\prime}$ whenever $U\left(1, \phi^{\prime}\right)= \pm 1$. (Compare the generalized gradient theorem.) 
we have $p-1>q-1$ and $p-1 \geqq 1-q$, so $p-1 \geqq|q-1|$. This furnishes (37). In case $n \geqq 3, F(\phi)$ is even positive since $(p-1)^{3}-(q-1)^{3}>0$ and the Cesàro means mentioned are positive if $k \geqq 2$. In this case $g(\nu \pi / n)>0$ either for all even $\nu$ or for all odd $\nu$.

Now,

$$
\begin{aligned}
G= & 2 \sum_{m=1}^{n}\left(p^{m}-q^{m}\right)\left(a_{m} \cos (\alpha-m \alpha / n)-b_{m} \sin (\alpha-m \alpha / n)\right) \\
& +\delta\left\{\left(p^{n}-q^{n}\right) a_{0}+2 \sum_{m=1}^{n}\left(p^{n-m}-q^{n-m}\right)\left(a_{m} \cos (m \alpha / n)+b_{m} \sin (m \alpha / n)\right)\right\} \\
= & \cos \alpha\{U(p, \alpha / n)-U(q, \alpha / n)\}+\sin \alpha\{V(p, \alpha / n)-V(q, \alpha / n)\} \\
& +\delta\left\{p^{n} U\left(p^{-1}, \alpha / n\right)-q^{n} U\left(q^{-1}, \alpha / n\right)\right\} ;
\end{aligned}
$$

so an application of Theorem A proves (36).

5 . If $n \geqq 3$ and the equality occurs in (36), $G$ will be equal to $\pm \lambda_{n}$ for proper choice of $\delta$. But, since $g(\nu \pi / n)>0$ either for all even $\nu$ or for all odd $\nu$, we must have $U(1, \nu \pi / n)=\gamma$ for the same $\nu$, where $\gamma$ represents either +1 or -1 .

The inverse statement is also clear. Assuming $U(1, \nu \pi / n)=\gamma$ for even $\nu$ we choose $\delta=+1$. Then (for $n \geqq 3$ ) $g(\nu \pi / n)=0, \nu$ odd, so $G=\gamma \lambda_{n}$ holds. This means that the equality holds in (36) since

$$
\begin{aligned}
|G| \leqq \mid \cos \alpha\{U(p, \alpha / n)-U(q, \alpha / n)\} & +\sin \alpha\{V(p, \alpha / n)-V(q, \alpha / n)\} \mid \\
& +\left|p^{n} U\left(p^{-1}, \alpha / n\right)-q^{n} U\left(q^{-1}, \alpha / n\right)\right| \leqq \lambda_{n} .
\end{aligned}
$$

In case $n=1, F(\phi)=\frac{1}{2}(p-q)>0$ so the same conditions of equality hold. This is true also for $n=2$ since $F(\phi)=(p-q)\left\{\frac{1}{2}(p+q-2)+2 \cos ^{2}(\phi / 2)\right\}$, the only exception being the case $p+q=2$ when at the same time $\alpha$ is an integral multiple of $\pi$. [For the calculation of $g(\nu \pi / 2)$ we need only $F\left(\frac{1}{2}(\alpha-\nu \pi)\right)$, which is 0 only if $p+q=2$ and $(\alpha-\nu \pi) / 2$ is an odd multiple of $\pi$.]

Regarding the special case $p>1, q=1$, see the joint paper of Rogosinski and Szegö in Mathematische Zeitschrift, vol. 28 (1928), pp. 73-94, in particular p. 81, (39).

6. The dual of III is:

IV. Let $U(r, \phi)$ be a harmonic polynomial and let $U(1, \phi)=U(\phi)$ belong to the class $L_{n}$. Then if $p>q \geqq 0, p+q \geqq 2$,

$$
\begin{aligned}
\mid \cos \alpha\{U(p, \alpha / n)-U(q, \alpha / n)\}+ & \sin \alpha\{V(p, \alpha / n)-V(q, \alpha / n)\} \mid \\
& \leqq p^{n} U\left(p^{-1}, \alpha / n\right)-q^{n} U\left(q^{-1}, \alpha / n\right) .
\end{aligned}
$$

The equality occurs if and only if $U(1, \nu \pi / n)$ vanishes either for all even $\nu$ or for all odd $\nu$, the only exception being the case $n=2, p+q=2, \alpha / \pi$ an integer.

From this follows: 
IV'. Let $U(r, \phi)$ be a harmonic polynomial of degree $n$ satisfying $U(1, \phi)$ $\geqq 0, \phi$ arbitrary real. Then if $p>q \geqq 0$ and $p+q \geqq 2$,

$$
\begin{aligned}
& {\left[\{U(p, \phi)-U(q, \phi)\}^{2}+\{V(p, \phi)-V(q, \phi)\}^{2}\right]^{1 / 2} } \\
& \leqq p^{n} U\left(p^{-1}, \phi\right)-q^{n} U\left(q^{-1}, \phi\right) .
\end{aligned}
$$

The equality occurs if and only if $U(r, \phi) \equiv \lambda\left\{1+r^{n} \cos n\left(\phi-\phi_{0}\right)\right\}, \lambda$ and $\phi_{0}$ real, $\lambda \geqq 0$, the only exception being the case $n=2, p+q=2$.

To prove IV we set

$$
\begin{aligned}
& \lambda_{m}=\left(p^{n-m}-q^{n-m}\right) \cos (m \alpha / n)+\delta\left(p^{m}-q^{m}\right) \cos (\alpha-m \alpha / n), \\
& \mu_{m}=\left(p^{n-m}-q^{n-m}\right) \sin (m \alpha / n)-\delta\left(p^{m}-q^{m}\right) \sin (\alpha-m \alpha / n)
\end{aligned}
$$

where as before, $\delta= \pm 1$. In virtue of the remark of $\$ 2.6$, it is only necessary to compute the corresponding quantity $G$, that is,

$$
\begin{aligned}
G= & \left(p^{n}-q^{n}\right) a_{0}+2 \sum_{m=1}^{n}\left(p^{n-m}-q^{n-m}\right)\left(a_{m} \cos (m \alpha / n)+b_{m} \sin (m \alpha / n)\right) \\
& +2 \delta \sum_{m=1}^{n}\left(p^{m}-q^{m}\right)\left(a_{m} \cos (\alpha-m \alpha / n)-b_{m} \sin (\alpha-m \alpha / n)\right)
\end{aligned}
$$

By Theorem B, we have $G \geqq 0$; or

$$
\begin{aligned}
& p^{n} U\left(p^{-1}, \alpha / n\right)-q^{n} U\left(q^{-1}, \alpha / n\right)+\delta \cos \alpha\{U(p, \alpha / n)-U(q, \alpha / n)\} \\
& +\delta \sin \alpha\{V(p, \alpha / n)-V(q, \alpha / n)\} \geqq 0 .
\end{aligned}
$$

7. The dual of the generalized gradient theorem is the following:

V. Let $U(r, \phi)$ be a harmonic polynomial of degree $n$ satisfying $U(1, \phi) \geqq 0$. Then for $r \leqq 1$,

$$
|\operatorname{grad} U(r, \phi)| \leqq n \sigma_{n}(r, \phi) .
$$

The equality occurs if and only if $U(r, \phi) \equiv \lambda\left\{1+r^{n} \cos n\left(\phi-\phi_{0}\right)\right\}, \lambda$ and $\phi_{0}$ real, $\lambda \geqq 0$. The only exception is the case in which $U\left(1, \phi_{0}\right)=0$; then the equality holds in (39) for $r=1, \phi=\phi_{0}$.

Incidentally $\mathrm{V}$ may be obtained by dividing each side of $\left(38^{\prime}\right)$ by $p-q$ and letting $p \rightarrow 1, q \rightarrow 1$. The left-hand side approaches $\left[\left\{U_{r}(1, \phi)\right\}^{2}\right.$ $\left.+\left\{V_{r}(1, \phi)\right\}^{2}\right]^{1 / 2}$, while the right-hand side approaches $n \sigma_{n}(1, \phi)$. This shows that (39) is true for $r=1$. But then, by a familiar application of the maximum principle it follows for $r<1$.

8. In terms of rational polynomials, III' may be stated in the following equivalent form:

VI. Let $F(z)=\sum_{m=0}^{n} c_{m} z^{m}$ be a polynomial of degree $n$ satisfying $|\Re F(z)| \leqq 1$ 
in $|z| \leqq 1$. If $p$ and $q$ are real numbers, $p>q \geqq 0, p+q \geqq 2$, then for $|z| \leqq 1$,

$$
|F(p z)-F(q z)|+\left|\Re\left\{p^{n} F\left(p^{-1} z\right)-q^{n} F\left(q^{-1} z\right)\right\}\right| \leqq p^{n}-q^{n} .
$$

The equality holds if and only if $F(z) \equiv a z^{n}+b$ where $|a|+|\Re b|=1$, the only exception being the case $n=2, p+q=2\left(^{5}\right)$.

Finally we observe that from the fact that (37) is non-negative the following refinement of a theorem of Rogosinski-Szegö follows, cf. loc. cit., p. 77, (21):

Let $\left\{s_{n}(z)\right\}$ be the partial sums of a power series $f(z)=c_{0}+c_{1} z+c_{2} z^{2}+\cdots$ convergent in the unit circle $|z|<1$ and satisfying the condition $|f(z)| \leqq 1$ for $|z|<1$. Then

$$
\left|\frac{r_{1}^{-n} s_{n}\left(r_{1} z\right)-r_{2}^{-n} s_{n}\left(r_{2} z\right)}{r_{1}^{-n}-r_{2}^{-n}}\right| \leqq 1
$$

where $|z| \leqq 1, r_{1}>0, r_{2}>0, r_{1} \neq r_{2}, r_{1}^{-1}+r_{2}^{-1} \geqq 2$.

9. Let

$$
F(z)=\sum_{m=0}^{n} c_{m} z^{m}
$$

be a polynomial of degree $n$ satisfying the condition $|\Re F(z)| \leqq 1$ in $|z| \leqq 1$; according to the gradient theorem

$$
\left|F^{\prime}(z)\right|=\left|\sum_{m=1}^{n} m c_{m} z^{m-1}\right| \leqq n, \quad|z| \leqq 1 .
$$

By repeated application of this theorem we obtain

$$
\left|F^{(k)}(z)\right| \leqq \frac{n !}{(n-k) !}
$$

or:

$$
\left|\sum_{m=k}^{n} C_{m, k} C_{m} z^{m-k}\right| \leqq C_{n, k} ; \quad|z| \leqq 1,1 \leqq k \leqq n .
$$

In all these inequalities the sign $<$ holds unless $F(z) \equiv \epsilon z^{n},|\epsilon|=1$.

Using (41) we now prove:

VII. Let $\gamma_{0}, \gamma_{1}, \cdots, \gamma_{n}$ be real numbers satisfying

$$
\delta \nu=\sum_{m=0}^{\nu}(-1)^{\nu+m} C_{\nu, m} \gamma_{m} \geqq 0, \quad 0 \leqq \nu \leqq n .
$$

If $F(z)=\sum_{m=0}^{n} c_{m} z^{m}$ is a polynomial of degree $n$ with real or complex coefficients

(5) See the footnote to III'. 
satisfying $|\Re F(z)| \leqq 1$ in $|z| \leqq 1$ we have

$$
\left|\sum_{m=0}^{n} \gamma_{m} c_{m} z^{m}\right| \leqq \gamma_{n}-\delta_{0}(1-|F(z)|), \quad|z| \leqq 1 .
$$

The equality holds if and only if $F(z) \equiv \epsilon z^{n},|\epsilon|=1,|z|=1$, or $\gamma_{0}=\gamma_{1}=\gamma_{2}$ $=\cdots=\gamma_{n}=\gamma$.

If $\delta_{\nu}$ is defined by (42) the known inversion formula

$$
\gamma_{m}=\sum_{\nu=0}^{m} C_{m, \nu} \delta_{\nu}
$$

may be proved in the following way. Expanding $e^{z}$ and using Cauchy multiplication we verify that

$$
\sum_{m=0}^{\infty}(-1)^{m} \frac{\delta_{m}}{m !} z^{m}=e^{z} \sum_{m=0}^{\infty}(-1)^{m} \frac{\gamma_{m}}{m !} z^{m} .
$$

Bringing $e^{z}$ to the left and again using Cauchy multiplication we obtain (44). Then (44) implies that

$$
\sum_{m=0}^{n} \gamma_{m} c_{m} z^{m}=\sum_{\nu=0}^{n} \delta_{\nu} z^{\nu}\left\{\sum_{m=\nu}^{n} C_{m, \nu} c_{m} z^{m-\nu}\right\}
$$

so, according to (41),

$$
\left|\sum_{m=0}^{n} \gamma_{m} c_{m} z^{m}\right| \leqq \delta_{0}|F(z)|+\sum_{\nu=1}^{n} \delta_{\nu}|z|^{\nu} C_{n, \nu} \leqq \delta_{0}|F(z)|+\gamma_{n}-\delta_{0}
$$

in $|z| \leqq 1$. If the equality holds, then $F(z) \equiv \epsilon z^{n},|\epsilon|=1,|z|=1$; or $\delta_{1}=\delta_{2}$ $=\cdots=\delta_{n}=0$. The second possibility is equivalent to the relation $\gamma_{0}=\gamma_{1}=\cdots=\gamma_{n}=\gamma$.

10. As a very simple application, (30) follows. Indeed, assuming that $U(r, \phi)$ is even, and defining $F(z)$ as in $\$ 3.6$, the coefficients $c_{m}$ are real. Putting $\gamma_{m}=R^{m}$, we obtain

$$
\delta_{\nu}=\sum_{m=0}^{\nu}(-1)^{\nu-m} R^{m} C_{\nu, m}=(R-1)^{\nu}>0 .
$$

Also $F(R)=U(R, 0)$, so from $(43)$ for $z=1$,

$$
|U(R, 0)|=\left|\sum_{m=0}^{n} c_{m} R^{m}\right| \leqq R^{n}-(1-|U(1,0)|) .
$$

This is slightly sharper than (30).

11. Another simple application follows by choosing $\gamma_{m}=p^{m}-q^{m}$ where $p>q \geqq 0$. Then $\delta_{\nu}=(p-1)^{\nu}-(q-1)^{\nu}$ so (42) is equivalent with $p+q \geqq 2$ pro- 
vided $n \geqq 2$. If $F(z)$ satisfies the conditions of VII it follows that

$$
|F(p)-F(q)| \leqq p^{n}-q^{n}
$$

This is slightly less general than VI.

12. Finally we point out that the following classical theorem of A. Markoff (cf. [2, pp. 359-360]) is also a simple consequence of the gradient theorem:

Let $h(x)$ be a polynomial of degree $n$ with real coefficients $\left(^{6}\right)$ satisfying the condition $|h(x)| \leqq 1$ in $-1 \leqq x \leqq 1$. Then $\left|h^{\prime}(x)\right|<n^{2}$ in $-1<x<1, n>1$. For $n=1$ we have $\left|h^{\prime}(x)\right| \leqq 1$.

Let

$$
h(\cos \phi)=\sum_{m=0}^{n} \alpha_{m} \cos m \phi, \quad F(z)=\sum_{m=0}^{n} \alpha_{m} z^{m} .
$$

We have for $0<\phi<\pi, z=e^{i \phi}$,

$$
\begin{aligned}
h^{\prime}(\cos \phi) & =\sum_{m=1}^{n} m \alpha_{m} \frac{\sin m \phi}{\sin \phi}=\sum_{m=1}^{n} m \alpha_{m}\left(z^{m}-\bar{z}^{m}\right) /(z-\bar{z}) \\
& =\frac{1}{z-\bar{z}} \int_{\bar{z}}^{z}\left\{\sum_{m=1}^{n} m^{2} \alpha_{m} \zeta^{m-1}\right\} d \zeta,
\end{aligned}
$$

the integration extending along the chord from $\bar{z}$ to $z$. Since $|\Re F(z)| \leqq 1$, $|z| \leqq 1$, we have $\left|F^{\prime}(z)\right| \leqq n$ and $\left|\left\{z F^{\prime}(z)\right\}^{\prime}\right| \leqq n^{2},|z| \leqq 1$, so for $n>1$, $\left|h^{\prime}(\cos \phi)\right|<n^{2}$ follows.

\section{Direct PROOF OF V}

1. Writing $U(\phi)$ and $V(\phi)$ for $U(1, \phi)$ and $V(1, \phi)$, respectively, to prove $V$ it will be suffcient to show that

$$
\left|U^{\prime}(0)+i V^{\prime}(0)\right| \leqq n \sigma_{n}(0)
$$

where $\sigma_{n}(0)$ is the $n$th Cesàro means of first order of $U(0)$. The proof of this inequality can be based on a theorem of Fejér which states: Let $U(\phi)$ be a trigonometric polynomial of degree $n$ which is non-negative for all real values of $\phi$. Then there exists a polynomial $A(z)=\sum_{\nu=0}^{n} a_{\nu} z^{\nu}$ such that $U(\phi)=\left|A\left(e^{i \phi}\right)\right|^{2}$.

In our case

and

$$
\begin{aligned}
U(\phi) & =\left|A\left(e^{i \phi}\right)\right|^{2}=\left|\sum_{\nu=0}^{n} a_{\nu} e^{i \nu \phi}\right|^{2} \\
& =\sum a_{\nu} \bar{a}_{\mu} \cos |\nu-\mu| \phi+i \sum a_{\nu} \bar{a}_{\mu} \operatorname{sgn}(\nu-\mu) \sin |\nu-\mu| \phi, \\
V(\phi) & =\sum a_{\nu} \bar{a}_{\mu} \sin |\nu-\mu| \phi-i \sum a_{\nu} \bar{a}_{\mu} \operatorname{sgn}(\nu-\mu) \cos |\nu-\mu| \phi
\end{aligned}
$$

$$
\begin{aligned}
U^{\prime}(0)+i V^{\prime}(0) & =i \sum(\nu-\mu) a_{\nu} \bar{a}_{\mu}+i \sum|\nu-\mu| a_{\nu} \bar{a}_{\mu}, \\
n \sigma_{n}(0) & =\sum(n-|\nu-\mu|) a_{\nu} \bar{a}_{\mu} .
\end{aligned}
$$

In these and the following expressions the summation extends over the range $0 \leqq \nu \leqq n, 0 \leqq \mu \leqq n$.

( $)$ This is not an essential restriction. 
The quadratic form with coefficients $|\nu-\mu|$ was studied by Szegö [6]. He showed that the second sum in (46) may be written in the form

$$
\sum|\nu-\mu| a_{\nu} \bar{a}_{\mu}=(n / 2)\left|\sum_{\nu=0}^{n} a_{\nu}\right|^{2}-(2 / n) \sum_{m=1}^{n} \frac{\left|\sum_{\nu-0}^{n} a_{\nu} z_{m}\right|^{2}}{\left|1-z_{m}\right|^{2}}
$$

where $z_{m}=e^{i(2 m-1) \pi / n}$ is a root of the equation $z^{n}+1=0$. Thus the linear transformation

$$
\begin{aligned}
A_{0} & =(n / 2)^{1 / 2}\left(a_{0}+a_{1}+\cdots+a_{n}\right)=(n / 2)^{1 / 2} A(1), \\
A_{m} & =(2 / n)^{1 / 2}\left(a_{0}+a_{1} z_{m}+\cdots+a_{n} z_{m}^{n}\right) /\left(1-z_{m}\right)=(2 / n)^{1 / 2} A\left(z_{m}\right) /\left(1-z_{m}\right), \quad 1 \leqq m \leqq n,
\end{aligned}
$$

furnishes the canonical representation

Also, from (47)

$$
\sum|\nu-\mu| a_{\nu} \bar{a}_{\mu}=\left|A_{0}\right|^{2}-\sum_{m=1}^{n}\left|A_{m}\right|^{2}
$$

$$
n \sigma_{n}(0)=2\left|A_{0}\right|^{2}-\left|A_{0}\right|^{2}+\sum_{m=1}^{n}\left|A_{m}\right|^{2}=\sum_{m=0}^{n}\left|A_{m}\right|^{2} .
$$

Next we express the first sum in (46) in terms of the $A_{m}$. Since

$$
\sum \nu a_{\nu} \bar{a}_{\mu}=\left(\sum_{\mu=0}^{n} \bar{a}_{\mu}\right)\left(\sum_{\nu=0}^{n} \nu a_{\nu}\right)=\overline{A(1)} A^{\prime}(1)
$$

and since $\sum \mu a_{\nu} \bar{a}_{\mu}$ is the conjugate of this,

$$
U^{\prime}(0)+i V^{\prime}(0)=-2 \Im\left\{\overline{A(1)} A^{\prime}(1)\right\}+i\left\{\left|A_{0}\right|^{2}-\sum_{m=1}^{n}\left|A_{m}\right|^{2}\right\} .
$$

Let $\omega(z)=\left(z^{n}+1\right)(z-1)$. Making use of (49), the Lagrange interpolation formula furnishes

$$
A(z)=(n / 2)^{1 / 2} \sum_{m=1}^{n} \frac{\omega(z) A_{m}\left(1-z_{m}\right)}{\left(z-z_{m}\right) \omega^{\prime}\left(z_{m}\right)}+(2 / n)^{1 / 2} A_{0} \frac{\omega(z)}{(z-1) \omega^{\prime}(1)},
$$

and this may be written

$$
A(z)=(2 n)^{-1 / 2} \sum_{m=1}^{n} A_{m} z_{m} \omega(z) /\left(z-z_{m}\right)+(2 n)^{-1 / 2} A_{0}\left(z^{n}+1\right)
$$

since $\omega^{\prime}\left(z_{m}\right)=n\left(1-z_{m}\right) / z_{m}, \omega^{\prime}(1)=2$. Differentiating (51) and setting $z=1$,

$$
A^{\prime}(1)=(2 / n)^{1 / 2} \sum_{m=1}^{n} A_{m} z_{m} /\left(1-z_{m}\right)+(n / 2)^{1 / 2} A_{0} .
$$

Using Cauchy's inequality

$$
\begin{aligned}
\left|-2 \Im\left\{\overline{A(1)} A^{\prime}(1)\right\}\right|^{2} & \leqq\left|(4 / n) \bar{A}_{0} \sum_{m=1}^{n} A_{m} z_{m} /\left(1-z_{m}\right)\right|^{2} \\
& \leqq\left(16 / n^{2}\right)\left|A_{0}\right|^{2}\left\{\sum_{m=1}^{n}\left|A_{m}\right|^{2}\right\}\left\{\sum_{m=1}^{n}\left|1-z_{m}\right|^{-2}\right\}=4\left|A_{0}\right|_{m=1}^{2}\left|A_{m}\right|^{2} .
\end{aligned}
$$

In the last equality the special case $a_{0}=1, a_{1}=a_{2}=\cdots=a_{n}=0$, of (48) is used in order to obtain $\sum_{m=1}^{n}\left|1-z_{m}\right|^{-2}$. From this

(54) $\left|U^{\prime}(0)+i V^{\prime}(0)\right| \leqq\left\{4\left|A_{0}\right|^{2} \sum_{m=1}^{n}\left|A_{m}\right|^{2}+\left(\left|A_{0}\right|^{2}-\sum_{m=1}^{n}\left|A_{m}\right|^{2}\right)^{2}\right\}^{1 / 2}=\left|A_{0}\right|^{2}+\sum_{m=1}^{n}\left|A_{m}\right|^{2}$

follows, which is the statement. The equality holds if and only if (53) is an equality; that is, if and only if either $A_{0}=0$, or $A_{m}=\lambda /\left(1-z_{m}\right), m=1,2, \cdots, n, \lambda$ a constant and $\overline{A(1)}\left\{A^{\prime}(1)-\frac{1}{2} n A(1)\right\}$ pure imaginary. The second condition implies $A\left(z_{m}\right)=$ const., $m=1,2, \cdots, n$, so $A(z) \equiv p z^{n}+q$ where $p$ and $q$ are constants. The additional condition regard- 
ing $A(1)$ and $A^{\prime}(1)$ furnishes $|p|^{2}=|q|^{2}$, so $A(z) \equiv p\left(z^{n}+\gamma\right),|\gamma|=1$. The other condition for equality, namely $A_{0}=0$, is equivalent with $U(0)=U(1,0)=0$. This establishes the statement.

2. The generalized gradient theorem is an immediate consequence of $\mathrm{V}$. If $U(r, \phi)$ satisfies the conditions of that theorem, then $1+\delta U(r, \phi)$ is non-negative in the unit circle where $\delta=+1$ or -1 . Thus by what we have just shown

$$
n+\delta n \sigma_{n}(r, \phi) \geqq|\operatorname{grad} U(r, \phi)| \text {. }
$$

Here $1+\delta \sigma_{n}(r, \phi)$ is the Cesàro means of the harmonic polynomial $1+\delta U(r, \phi)$.

\section{Part II. Three-dimensional harmonic polynomials}

\section{INTRODUCTION}

In this part we deal with harmonic polynomials $u(x, y, z)$, that is, polynomials in the cartesian coordinates $x, y, z$ satisfying Laplace's differential equation $\Delta u=0$. We frequently prefer to use polar coordinates $r, \theta, \phi$ (in the usual notation) writing: $u(x, y, z)=U(r, \theta, \phi)$. Then the standard representation

$$
\begin{aligned}
U(r, \theta, \phi)= & \sum_{\mu=0}^{n} r^{\mu}\left\{a_{\mu 0} P_{\mu}(\cos \theta)\right. \\
& \left.+\sum_{\nu=1}^{\mu} \sin ^{\nu} \theta P_{\mu}^{(\nu)}(\cos \theta)\left(a_{\mu \nu} \cos \nu \phi+b_{\mu \nu} \sin \nu \phi\right)\right\}
\end{aligned}
$$

holds; here $P_{n}(x)$ is the $n$th polynomial of Legendre in the customary notation.

1. As already mentioned, our main task in Part II is to prove the threedimensional analogue of inequality (30) of Part I, that is:

Let $u(x, y, z)$ be an arbitrary harmonic polynomial of degree $n$ satisfying the condition

$$
|U(x, y, z)| \leqq 1, \quad x^{2}+y^{2}+z^{2} \leqq 1 .
$$

Let $n \geqq 1$. Then at a point $(x, y, z)$ in the exterior of the unit sphere, that is, for $x^{2}+y^{2}+z^{2}=R^{2}>1$,

$$
\begin{aligned}
|u(x, y, z)| \leqq & \frac{1}{2} \frac{2 \cdot 4 \cdots 2 n}{3 \cdot 5 \cdots(2 n+1)}\left\{(2 n+1) R^{n}\right. \\
& +(2 n-3) \frac{n^{2}-(n+1)^{2}}{n^{2}-(n-2)^{2}} R^{n-2} \\
& +(2 n-7) \frac{n^{2}-(n+1)^{2}}{n^{2}-(n-2)^{2}} \frac{n^{2}-(n-1)^{2}}{n^{2}-(n-4)^{2}} R^{n-4} \\
& +\cdots\}=c_{n}(R) .
\end{aligned}
$$


This bound $c_{n}(R)$ is attained exclusively for the polynomials $u(x, y, z)$ whose boundary values on the unit sphere appear in the form $\pm \cos n \gamma$ where $\gamma$ is the spherical distance of the variable point $(1, \theta, \phi)$ from a fixed point $\left(1, \theta_{0}, \phi_{0}\right)$.

For the polynomials mentioned the bound $c_{n}(R)$ is attained only when $(x, y, z)$ lies on the line connecting $\left(1, \theta_{0}, \phi_{0}\right)$ with the origin.

A direct characterization of $c_{n}(R)$ is the following: Write $\cos n \theta$ as a linear combination of Legendre functions $P_{\nu}(\cos \theta)$ :

$$
\cos n \theta=h_{n 0} P_{0}(\cos \theta)+h_{n 1} P_{1}(\cos \theta)+\cdots+h_{n n} P_{n}(\cos \theta)
$$

Then

$$
c_{n}(R)=h_{n 0}+h_{n 1} R+\cdots+h_{n n} R^{n},
$$

so $c_{n}(R)$ is a polynomial of degree $n$ in $R$.

It is easy to show $(\$ 7)$ that for $R>1$

$$
c_{n}(R) \simeq \frac{1}{2} \pi^{1 / 2}\left(1-R^{-2}\right)^{1 / 2} n^{1 / 2} R^{n} \quad \text { as } n \rightarrow \infty,
$$

so the result is slightly different in character from the corresponding result in the two-dimensional case in which the maximum in question is precisely $R^{n}$.

The proof of the Theorem mentioned requires a rather elaborate apparatus, in particular a discussion of the trigonometric polynomials

$$
c_{n}(R)+2 \sum_{m=1}^{n-1} c_{n-m}(R) \cos m \theta+c_{0}(R) \cos n \theta .
$$

This discussion is made possible by the following remarkable representation of $c_{n}(R)$ :

$$
\begin{aligned}
& c_{n}(R)=R^{n}+\int_{-1 \leqq t \leqq 0,1 \leqq t \leqq R} \frac{\left(R^{2}-1\right)\left(t^{2}-1\right)}{4 t^{2}} \\
& \cdot\left\{\frac{t}{(R-t)(R t-1)}\right\}^{3 / 2}\left(R^{n}-t^{n}\right) d t, \quad R>1 .
\end{aligned}
$$

We prove (8) by function-theoretic considerations applied to the "generating function" of the sequence $\left\{c_{n}(R)\right\}$.

2. In order to prove (3), say for $\theta=0$, we might try to use an argument similar to that in $\$ 4.10$ of Part I. Since

$$
U(R, \theta, \phi)=\sum_{m=0}^{n} \frac{(R-1)^{m}}{m !}\left\{\frac{\partial^{m} U(r, \theta, \phi)}{\partial r^{m}}\right\}_{r=1},
$$

it would suffice to show that all the expressions in the braces \{\} assume their maximum value if $U(1, \theta, \phi)=\cos n \theta$. This is indeed the case for $m=0$, and, according to a recent result of Szegö [7], also for $m=1$. In $\S 6$ of Part II 
we shall prove this for $m=2$; however the proof (or refutation) of the general assertion seems to be difficult.

- The theorem of Szegö just referred to states even more generally that under condition (2) the directional derivatives $|\partial u / \partial l|$ where $\left(x^{2}+y^{2}+z^{2}\right)^{1 / 2}$ $=r=1$ and $l$ is an arbitrary direction, assume their maximum value only for functions with boundary values of the form $\pm \cos n \gamma$. [This is of course equivalent to an estimate of $|\operatorname{grad} u|$.] More generally, we might ask for the maximum of

$$
\left|\frac{\partial}{\partial l_{1}} \frac{\partial}{\partial l_{2}} \cdots \frac{\partial}{\partial l_{m}} u\right|, \quad r=1
$$

where $l_{1}, l_{2}, \cdots, l_{m}$ denote arbitrary directions. The former problem is a special case corresponding to coinciding directions $l_{1}, l_{2}, \cdots, l_{m}$.

\section{Preliminaries}

1. Considering the set of harmonic polynomials of degree $n$ which satisfy condition (2), we see that the maximum of $|u|$ at an exterior point $(x, y, z)$ is the same at all points on a sphere with center at the origin. Therefore we assume that $x=y=0, z=R$. Also, by taking the mean-value

$$
(2 \pi)^{-1} \int_{-\pi}^{\pi} U(r, \theta, \phi) d \phi
$$

we see that the maximum remains the same by restricting the set in question to the polynomials $u(x, y, z)=U(r, \theta, \phi)$ independent of $\phi$. This leads to the following equivalent formulation of the problem:

Let $u(\theta)$ be a cosine polynomial of degree $n$,

$$
u(\theta)=\sum_{m=0}^{n} \alpha_{m} P_{m}(\cos \theta)
$$

expanded in terms of Legendre polynomials, and let $|u(\theta)| \leqq 1$ for $0 \leqq \theta \leqq \pi$. What is the maximum of $\left|\sum_{m=0}^{n} \alpha_{m} R^{m}\right|$ ?

2. Since

$$
\alpha_{m}=\frac{1}{2}(2 m+1) \int_{0}^{\pi} u(\theta) P_{m}(\cos \theta) \sin \theta d \theta
$$

we have

$$
\sum_{m=0}^{n} \alpha_{m} R^{m}=\int_{0}^{\pi} u(\theta)\left\{\sum_{m=0}^{n} \frac{1}{2}(2 m+1) P_{m}(\cos \theta) R^{m}\right\} \sin \theta d \theta .
$$

We shall prove that the maximum in question is attained when $u(\theta) \equiv \pm \cos n \theta$ 
and that this maximum is

$$
c_{n}(R)=\int_{0}^{\pi} \cos n \theta\left\{\sum_{m=0}^{n} \frac{1}{2}(2 m+1) P_{m}(\cos \theta) R^{m}\right\} \sin \theta d \theta .
$$

This will imply (5). Representation (3) is obtained by taking into account the well known expression of $\cos n \theta$ in terms of Legendre polynomials $\left({ }^{7}\right)$.

3. The polynomial $c_{n}(R)$ contains only terms $R^{n}, R^{n-2}, R^{n-4}, \cdots$, the first coefficient being positive and all the remaining coefficients being negative. Since $c_{n}(1)=1$, this implies, as is easily seen, that all derivatives of $c_{n}(R)$ are positive when $R \geqq 1$. We have

$$
\begin{array}{ll}
c_{0}(R)=1, \quad c_{1}(R)=R, & c_{2}(R)=\frac{4}{3} R^{2}-\frac{1}{3}, \\
c_{3}(R)=\frac{8}{5} R^{3}-\frac{3}{5} R, & c_{4}(R)=\frac{64}{35} R^{4}-\frac{16}{21} R^{2}-\frac{1}{15} .
\end{array}
$$

2. Generating Function of the SEQUence $\left\{c_{n}(R)\right\}$

1. The following important result is to be proved.

Let $R>1$. The generating function of the sequence $\left\{c_{n}(R)\right\}$ is, for $|z|<R^{-1}$,

$$
\begin{aligned}
F(R, z)= & F(z)=\frac{1}{2} c_{0}(R)+\sum_{n=1}^{\infty} c_{n}(R) z^{n} \\
= & \frac{1}{2}\left(R^{2}-1\right) \frac{1-z^{2}}{\{(R-z)(1-R z)\}^{3 / 2}} z^{1 / 2} \arctan \left(\frac{z(R-z)}{1-R z}\right)^{1 / 2} \\
& +\frac{1}{2} R \frac{1-z^{2}}{(R-z)(1-R z)} .
\end{aligned}
$$

The determination of the multi-valued functions involved in this formula is evident: for small positive $z$ we take $\{(R-z)(1-R z)\}^{3 / 2}>0, z^{1 / 2}>0$, $\{z(R-z) /(1-R z)\}^{1 / 2}>0$, and the branch of arc $\tan \omega$ which vanishes at $\omega=0$.

The starting point of the proof is definition (12) of $c_{n}(R)$. We make use of Legendre functions of the second kind (cf. Szegö [7, p. 58]).

2. Let $Q_{n}(\xi)$ be Legendre functions of the second kind in the usual notation. It is well known that

$$
\lim _{\epsilon \rightarrow+0}\left\{Q_{n}(u+i \epsilon)-Q_{n}(u-i \epsilon)\right\}=-i \pi P_{n}(u),-1<u<1,
$$

so if $z=r e^{i \theta}, 0<r<1,0<\theta<\pi$, then

$$
P_{n}(\cos \theta)=\frac{2}{\pi} \lim _{r \rightarrow 1-0} \Im Q_{n}\left(\frac{1}{2}\left(z+z^{-1}\right)\right) .
$$

( $\left.{ }^{7}\right)$ See E. W. Hobson, The Theory of Spherical and Ellipsoidal Harmonics, Cambridge University Press, 1931; p. 47, (48). 
Hence

$\sin \theta \sum_{m=0}^{n} \frac{1}{2}(2 m+1) P_{m}(\cos \theta) R^{m}$

$$
\begin{aligned}
& =\lim _{r \rightarrow 1-0} \frac{z-z^{-1}}{2 i} \frac{2}{\pi} \Im\left\{\sum_{m=0}^{n} \frac{1}{2}(2 m+1) Q_{m}\left(\frac{1}{2}\left(z+z^{-1}\right)\right) R^{m}\right\} \\
& =\lim _{r \rightarrow 1-0} \Re\left\{\frac{1-z^{2}}{\pi z} \sum_{m=0}^{n} \frac{1}{2}(2 m+1) Q_{m}\left(\frac{1}{2}\left(z+z^{-1}\right)\right) R^{m}\right\} .
\end{aligned}
$$

$$
Q_{m}\left(\frac{1}{2}\left(z+z^{-1}\right)\right)
$$

can be expanded into a power series of $z$ convergent for $|z|<1$; the first nonvanishing term of this series is $c z^{m+1}, c \neq 0$. Also $\left(^{8}\right)$

$$
\left|Q_{m}\left(\frac{1}{2}\left(z+z^{-1}\right)\right)\right|^{1 / m} \simeq|z|, \quad|z|<1, m \rightarrow \infty,
$$

so the series

$$
\sum_{m=0}^{\infty} \frac{1}{2}(2 m+1) Q_{m}\left(\frac{1}{2}\left(z+z^{-1}\right)\right) R^{m}
$$

is convergent for $|z| \cdot R<1$.

According to (12) and (17), $c_{n}(R)$ is the real part of

$$
\lim _{r \rightarrow 1-0} \frac{2}{\pi} \int_{0}^{\pi} \cos n \theta \cdot\left\{\frac{1-z^{2}}{2 z} \sum_{m=0}^{n} \frac{1}{2}(2 m+1) Q_{m}\left(\frac{1}{2}\left(z+z^{-1}\right)\right) R^{m}\right\} d \theta .
$$

This is simply the coefficient of $z^{n}$ in the expansion of the function of $z$ in the curled brackets. [For $n=0$ this gives twice the constant term of this expansion.] However, this coefficient does not change if the sum $\sum_{m=0}^{n}$ is replaced by $\sum_{m=0}^{\infty}$; so we obtain for the generating function (14) the representation

$$
F(R ; z)=\frac{1-z^{2}}{2 z} \sum_{m=0}^{\infty} \frac{1}{2}(2 m+1) Q_{m}\left(\frac{1}{2}\left(z+z^{-1}\right)\right) R^{m} .
$$

3. In order to calculate the last expression, we use the formula $\left({ }^{9}\right)$

$$
Q_{m}\left(\frac{1}{2}\left(z+z^{-1}\right)\right)=\frac{1}{2} \int_{-\infty}^{+\infty}\left\{\frac{1}{2}\left(z+z^{-1}\right)+\frac{1}{2}\left(z^{-1}-z\right) \cosh \tau\right\}^{-m-1} d \tau
$$

For sake of simplicity we assume that $z$ is real and positive, $0<z<R^{-1}$. Since

$$
\frac{1}{2}\left(z+z^{-1}\right)+\frac{1}{2}\left(z^{-1}-z\right) \cosh \tau \geqq \frac{1}{2}\left(z+z^{-1}\right)+\frac{1}{2}\left(z^{-1}-z\right)=z^{-1}>R,
$$

(8) Cf. for instance, G. Szegö, Orthogonal Polynomials, American Mathematical Society Colloquium Publications, vol. 23, 1939, p. 195, (8.23.2).

(9) See, for instance, Szegö, loc. cit., p. 90, (4.81.2). 
term-by-term integration is permitted and we obtain

$$
\begin{aligned}
F(R ; z) & =\frac{1-z^{2}}{8 z} \int_{-\infty}^{+\infty} \sum_{m=0}^{\infty}(2 m+1)\left\{\frac{1}{2}\left(z+z^{-1}\right)+\frac{1}{2}\left(z^{-1}-z\right) \cosh \tau\right\}^{-m-1} R^{m} d \tau \\
& =\frac{1-z^{2}}{8 z} \int_{-\infty}^{+\infty} \frac{\frac{1}{2}\left(z+z^{-1}\right)+R+\frac{1}{2}\left(z^{-1}-z\right) \cosh \tau}{\left\{\frac{1}{2}\left(z+z^{-1}\right)-R+\frac{1}{2}\left(z^{-1}-z\right) \cosh \tau\right\}^{2}} d \tau \\
& =\frac{1}{4} \int_{-\infty}^{+\infty} \frac{A+\cosh \tau}{(B+\cosh \tau)^{2}} d \tau=\int_{1}^{\infty} \frac{1+t^{2}+2 A t}{\left(1+t^{2}+2 B t\right)^{2}} d t
\end{aligned}
$$

where

$$
A=\frac{1+2 R z+z^{2}}{1-z^{2}}, \quad B=\frac{1-2 R z+z^{2}}{1-z^{2}} .
$$

Obviously $A>1,-1<B<+1$.

4. The primitive function of the last integrand in (21) is

$$
\frac{1-A B}{\left(1-B^{2}\right)^{3 / 2}} \arctan \left\{\frac{t+B}{\left(1-B^{2}\right)^{1 / 2}}\right\}+\frac{B-A}{1-B^{2}} \frac{B t+1}{1+t^{2}+2 B t} .
$$

Hence, omitting trivial details, we find that

$$
\begin{aligned}
F(R ; z)= & \frac{\left(R^{2}-1\right) 4 z^{2}\left(1-z^{2}\right)}{\{4 z(R-z)(1-R z)\}^{3 / 2}}\left[\frac{\pi}{2}-\arctan \left(\frac{1}{z} \frac{1-R z}{R-z}\right)^{1 / 2}\right] \\
& +\frac{2 R z\left(1-z^{2}\right)}{4 z(R-z)(1-R z)},
\end{aligned}
$$

which easily furnishes (14).

Another way of writing (14) is

$$
\begin{aligned}
F(R ; z)= & \frac{1}{4 i}\left(R^{2}-1\right) \frac{1-z^{2}}{\left\{(R-z)(1-R z)^{3 / 2}\right.} z^{1 / 2} \log \frac{1+i\left(\frac{z(R-z)}{1-R z}\right)^{1 / 2}}{1-i\left(\frac{z(R-z)}{1-R z}\right)^{1 / 2}} \\
& +\frac{1}{2} R \frac{1-z^{2}}{(R-z)(1-R z)} \\
= & G(R ; z)+\frac{1}{2} R \frac{1-z^{2}}{(R-z)(1-R z)} .
\end{aligned}
$$

Obviously

$$
G(R ; z)=G(z)=\sum_{n=1}^{\infty}\left\{c_{n}(R)-\frac{1}{2}\left(R^{n}+R^{-n}\right)\right\} z^{n}
$$




\section{Discussion OF THE FUNCTION $G(z)$}

The function $G(R ; z)=G(z)$ defined by $\left(14^{\prime}\right)$ and $(23)$ is regular except perhaps at the points

$$
-1,0, R^{-1}, 1, R, \infty .
$$

We intend to discuss the behavior of $G(z)$ near these points; also, we wish to calculate the limits of $G(z)$. on the "upper" and "lower" border of the real segments $[-\infty,-1]$ and $\left[R^{-1}, 1\right]$.

1. $-\infty<z<-1$. First, for $-1<z<0$ we have

$$
G(z)=\frac{1}{4}\left(R^{2}-1\right) \frac{1-z^{2}}{\{(R-z)(1-R z)\}^{3 / 2}}(-z)^{1 / 2} \log \frac{1-\left(\frac{-z(R-z)}{1-R z}\right)^{1 / 2}}{1+\left(\frac{-z(R-z)}{1-R z}\right)^{1 / 2}} .
$$

Here \{\}$^{3 / 2}>0,(-z)^{1 / 2}>0,(-z(R-z) /(1-R z))^{1 / 2}>0$, and the log is real. This easily furnishes, for $-\infty<z<-1$,

$$
G(z)=\frac{1}{4}\left(R^{2}-1\right) \frac{1-z^{2}}{\{(R-z)(1-R z)\}^{3 / 2}}(-z)^{1 / 2}\left\{\log \frac{\left(\frac{-z(R-z)}{1-R z}\right)^{1 / 2}-1}{\left(\frac{-z(R-z)}{1-R z}\right)^{1 / 2}+1} \pm i \pi\right\}
$$

where the signs + and - refer to the upper and lower border, respectively.

2. $R^{-1}<z<1$. First, for $0<z<R^{-1}$ we have the formula $\left(14^{\prime}\right)$ in which \{\}$^{3 / 2}>0, z^{1 / 2}>0,(z(R-z) /(1-R z))^{1 / 2}>0$, and the $\log$ vanishes for $z=0$. If $z$ increases from 0 to $R^{-1}$, the expression $z(R-z) /(1-R z)$ increases from 0 to $+\infty$ and the imaginary part of the log increases from 0 to $\pi$.

If $z$ describes a small circle around $R^{-1}$, from $(1-R z)^{3 / 2}, z<R^{-1}$, we obtain by analytic continuation $(R z-1)^{3 / 2} e^{\mp 3 i \pi / 2}, z>R^{-1}$, on the upper and lower border, respectively. Also, from

$$
i\left(\frac{z(R-z)}{1-R z}\right)^{1 / 2},
$$$$
z<R^{-1}
$$

we obtain $\mp(z(R-z) /(R z-1))^{1 / 2}, z>R^{-1}$. Thus we find for $z>R^{-1}$

$$
G(z)=\frac{1}{4 i}\left(R^{2}-1\right) e^{ \pm 3 i \pi / 2} \frac{1-z^{2}}{\{(R-z)(R z-1)\}^{3 / 2}} z^{1 / 2} \log \frac{1 \mp\left(\frac{z(R-z)}{R z-1}\right)^{1 / 2}}{1 \pm\left(\frac{z(R-z)}{R z-1}\right)^{1 / 2}}
$$

where the imaginary part of the log approaches $\pi$ as $z \rightarrow R^{-1}+0$. 
This can be written in the form

$$
G(z)=\frac{1}{4}\left(R^{2}-1\right) \frac{1-z^{2}}{\{(R-z)(R z-1)\}^{3 / 2}} z^{1 / 2}\left\{\log \frac{1+\left(\frac{R z-1}{z(R-z)}\right)^{1 / 2}}{1-\left(\frac{R z-1}{z(R-z)}\right)^{1 / 2}} \mp i \pi\right\}
$$

on the upper and lower border, respectively.

3. $1<z<R$. If $z$ increases from $R^{-1}$ to 1 , the expression $(R z-1) /(z(R-z))$ increases from 0 to 1 ; at $z=1$ the $\log$ becomes singular. Encircling $z=1$, we obtain

$$
\begin{aligned}
G(z) & =\frac{1}{4}\left(R^{2}-1\right) \frac{1-z^{2}}{\{(R-z)(R z-1)\}^{3 / 2}} z^{1 / 2} \log \frac{1+\left(\frac{R z-1}{z(R-z)}\right)^{1 / 2}}{\left(\frac{R z-1}{z(R-z)}\right)^{1 / 2}-1} \\
& =\frac{1}{4}\left(R^{2}-1\right) \frac{1-z^{2}}{\{(R-z)(R z-1)\}^{3 / 2}} z^{1 / 2} \log \frac{1+\left(\frac{z(R-z)}{R z-1}\right)^{1 / 2}}{1-\left(\frac{z(R-z)}{R z-1}\right)^{1 / 2}}
\end{aligned}
$$

on both borders. Here the log is real.

This shows that the branch of the function $G(z)$ which we have considered is single valued for $z>1$; it has a simple pole at $z=R$.

\section{INTEGRAL REPRESENTATION OF $c_{n}(R)$}

1. Since $G(0)=0$, we have for $n \geqq 1$

$$
c_{n}(R)-\frac{1}{2}\left(R^{n}+R^{-n}\right)=\frac{1}{2 \pi i} \int G(z)\left(z^{-n}-R^{n}\right) z^{-1} d z
$$

where the integration curve consists of the following parts:

(a) a "large" circle $|z|=\mathrm{P},|\operatorname{arc} z| \leqq \pi-\epsilon, \epsilon \rightarrow+0$;

(b) the segment $[-\mathrm{P},-1]$ described twice, the upper border in the increasing, the lower in the decreasing way;

(c) the segment $\left[R^{-1}, 1\right]$ described twice, the upper border in the increasing, the lower in the decreasing way;

(d) a "small" circle around $z=R$ described in the negative sense.

The circle in (d) furnishes the negative residue corresponding to $z=R$.

2. Since

$$
G(z)=O\left(|z|^{-1 / 2}\right) \text { as } z \rightarrow \infty,
$$

the contribution of the large circle in (a) vanishes as the radius $\mathrm{P}$ approaches $\infty$.

According to (24) we obtain as the contribution of $[-\infty,-1]$ : 


$$
\begin{aligned}
& \frac{1}{4}\left(R^{2}-1\right) \int_{-\infty}^{-1} \frac{1-z^{2}}{\{(R-z)(1-R z)\}^{3 / 2}}(-z)^{1 / 2}\left(z^{-n}-R^{n}\right) z^{-1} d z \\
& \quad=-\frac{1}{4}\left(R^{2}-1\right) \int_{0}^{-1} \frac{1-t^{-2}}{\left\{\left(R-t^{-1}\right)\left(1-R t^{-1}\right)\right\}^{3 / 2}}(-t)^{-1 / 2}\left(t^{n}-R^{n}\right) t^{-1} d t .
\end{aligned}
$$

The contribution of $\left[R^{-1}, 1\right]$ is, according to (25),

$$
\begin{aligned}
-\frac{1}{4}\left(R^{2}-1\right) & \int_{R^{-1}}^{1} \frac{1-z^{2}}{\{(R-z)(R z-1)\}^{3 / 2}} z^{1 / 2}\left(z^{-n}-R^{n}\right) z^{-1} d z \\
= & \frac{1}{4}\left(R^{2}-1\right) \int_{R}^{1} \frac{1-t^{-2}}{\left\{\left(R-t^{-1}\right)\left(R t^{-1}-1\right)\right\}^{3 / 2}} t^{-1 / 2}\left(t^{n}-R^{n}\right) t^{-1} d t .
\end{aligned}
$$

These integrals are convergent.

Around $z=R$, the function $G(z)$ is single valued, and from (26) we find

$$
\begin{aligned}
G(z) & =\frac{1}{4}\left(R^{2}-1\right) \frac{1-z^{2}}{\{(R-z)(R z-1)\}^{3 / 2}} z^{1 / 2} \cdot 2\left(\frac{z(R-z)}{R z-1}\right)^{1 / 2}+\cdots \\
& =\frac{1}{2}\left(R^{2}-1\right) \frac{\left(1-z^{2}\right) z}{(R z-1)^{2}} \frac{1}{R-z}+\cdots=\frac{1}{2} R(z-R)^{-1}+\cdots
\end{aligned}
$$

where the terms not written out are regular at $z=R$. Thus the contribution of $(d)$ is

$$
-\frac{1}{2} R\left(R^{-n}-R^{n}\right) R^{-1}=\frac{1}{2}\left(R^{n}-R^{-n}\right) .
$$

Recapitulating, we obtain the important result

$$
c_{n}(R)=R^{n}+\int_{-1 \leqq t \leqq 0,1 \leqq t \leqq R} p(R ; t)\left(R^{n}-t^{n}\right) d t,
$$

where

$$
p(R ; t)=p(t)=\frac{\left(R^{2}-1\right)\left(t^{2}-1\right)}{4 t^{2}}\left\{\frac{t}{(R-t)(R t-1)}\right\}^{3 / 2} .
$$

Formula (30) is trivial for $n=0$. We note that (except $t=0) \operatorname{sgn} p(R ; t)$ $=\operatorname{sgn}\left(t^{2}-1\right)$.

\section{Solution of the MaXimum PROBlem}

1. Returning again to the maximum problem formulated in $\$ 1$ of this part, we start with the remark that, as a generalization of (12),

$$
c_{\nu}(R)=\int_{0}^{\pi} \cos \nu \theta\left\{\sum_{m=0}^{n} \frac{1}{2}(2 m+1) P_{m}(\cos \theta) R^{m}\right\} \sin \theta d \theta, \quad 0 \leqq \nu \leqq n,
$$

holds. Indeed, 


$$
\int_{0}^{\pi} \cos \nu \theta P_{m}(\cos \theta) \sin \theta d \theta=0
$$

Therefore, the cosine expansion of the function $\pi \sum_{m=0}^{n} \frac{1}{2}(2 m+1) P_{m}(\cos \theta) R^{m}$ $\sin \theta$ begins with the terms

$$
c_{0}(R)+2 \sum_{\nu=1}^{n} c_{\nu}(R) \cos \nu \theta .
$$

Thus we obtain from (11)

$$
\sum_{m=0}^{n} \alpha_{m} R^{m}=\pi^{-1} \int_{0}^{\pi} u(\theta)\left\{c_{0}(R)+2 \sum_{\nu=1}^{n} c_{\nu}(R) \cos \nu \theta\right\} d \theta
$$

2. Application of Theorem A of Part I to the last expression shows that if

$$
C_{\nu n}(R)=\sum_{m=0}^{n}{ }^{\prime} c_{n-m}(R) \cos (m \nu \pi / n)>0, \quad 0 \leqq \nu \leqq n,
$$

then the maximum of (32) will be attained only by $u(\theta)= \pm \cos n \theta$. Here, as in Part I, the symbol $\sum_{m=0}^{\prime n}$ means that the terms $m=0$ and $m=n$ have to be multiplied by $\frac{1}{2}$. It is needless to say that $R>1$.

Our next purpose is to prove these inequalities. Note that having done this we have proved much more than the original statement, namely the inequality $\left|\sum_{m=0}^{n} \alpha_{m} R^{m}\right| \leqq c_{n}(R)$ under the condition that $|u(\nu \pi / n)| \leqq 1$, $0 \leqq \nu \leqq n$.

Since $c_{n}(R)>0$ (cf. $\$ 1.3$ ), it suffices to prove (33) for $1 \leqq \nu \leqq n$.

3 . We combine the representation (30) with formula (20) of Part I and find that

$$
\begin{aligned}
C_{\nu n}(R)= & \frac{1}{2}\left(R^{2}-1\right) \frac{R^{n}+(-1)^{\nu-1}}{1-2 R \cos (\nu \pi / n)+R^{2}} \\
& +\int p(R ; t)\left\{\frac{1}{2}\left(R^{2}-1\right) \frac{R^{n}+(-1)^{\nu-1}}{1-2 R \cos (\nu \pi / n)+R^{2}}\right. \\
& \left.-\frac{1}{2}\left(t^{2}-1\right) \frac{t^{n}+(-1)^{\nu-1}}{1-2 t \cos (\nu \pi / n)+t^{2}}\right\} d t
\end{aligned}
$$

where the integration extends over $-1 \leqq t \leqq 0$ and $1 \leqq t \leqq R$. Then to prove (33) it will be sufficient to show that

$$
1+\int p(R ; t)\left\{1-\frac{t^{2}-1}{R^{2}-1} \frac{t^{n}+(-1)^{\nu-1}}{R^{n}+(-1)^{\nu-1}} \frac{1-2 R \cos (\nu \pi / n)+R^{2}}{1-2 t \cos (\nu \pi / n)+t^{2}}\right\} d t>0
$$

We consider only the case $n \geqq 3$, since (33) may be proved directly for $n=1,2$. Now, let us consider the expression 


$$
-\left\{t^{n}+(-1)^{\nu-1}\right\} \frac{1-2 R \lambda+R^{2}}{1-2 t \lambda+t^{2}}=k(\lambda)
$$

as a function of $\lambda,-1 \leqq \lambda \leqq 1$. We have

$$
k^{\prime}(\lambda)=2(R-t)\left\{t^{n}+(-1)^{\nu-1}\right\} \frac{1-R t}{\left(1-2 t \lambda+t^{2}\right)^{2}} .
$$

Let $\nu$ be even. In this case $k^{\prime}(\lambda)<0$ whether $-1<t<0$ or $1<t<R$, so $\min k(\lambda)$ is attained for $\lambda=+1$. Since (35) is positive for $\nu=0$, it must be positive for all even values of $\nu$.

It remains to show that (35) is satisfied for odd $\nu$.

4. First let $1<t<R$. Since $\nu$ is odd,

$$
q(t)=\frac{\left(t^{2}-1\right)\left(t^{n}+1\right)}{1-2 t \cos (\nu \pi / n)+t^{2}}=\frac{\left(t^{2}-1\right)\left(t^{n}+1\right)}{\left(t-e^{i \nu \pi / n}\right)\left(t-e^{-i \nu \pi / n}\right)}
$$

is a polynomial of degree $n$ with positive highest coefficient, all of whose roots are on the unit circle $|t|=1$. (For $\nu=n$ the presence of the factor $t^{2}-1$ is essential, however not for $1 \leqq \nu \leqq n-1$.) Then $q(t)$ is a convex function for $t>1$, and it vanishes at $t=1$. Thus $0<q(t)<q(R)(t-1) /(R-1)$ for $1<t<R$. The expression in curled brackets \{\} in (35) is of the form $1-q(t) / q(R)$ and so it must be greater than $(R-t) /(R-1), 1<t<R$.

Thus the contribution of the range $1<t<R$ to the integral of (35) will be greater than

$$
\frac{R+1}{4} \int_{1}^{R} \frac{t^{2}-1}{t^{1 / 2}(R-t)^{1 / 2}(R t-1)^{3 / 2}} d t .
$$

Now,

$$
|(R-t)(R t-1)|^{1 / 2} \leqq \frac{1}{2}\left(R^{2}-1\right) R^{-1 / 2},|R t-1| \leqq R^{2}-1 \text { for } 1<t<R,
$$

so $(37)$ is greater than

$$
\frac{1}{2}(R+1)^{-1}(R-1)^{-2} \int_{1}^{R}\left(t^{2}-1\right) d t=\frac{1}{6}(R+2)(R+1)^{-1}>\frac{1}{6} .
$$

5. Let $-1<t<0$. Referring to (36), we see that in the present case $k(\lambda)$ is increasing (since $\nu$ is odd). Then, by what we have just shown, (35) will be a consequence of the following inequality

$$
\frac{7}{6}+\int_{-1}^{0} p(R ; t)\left\{1-\frac{t^{2}-1}{R^{2}-1} \frac{t^{n}+1}{R^{n}+1} \frac{(R+1)^{2}}{(t+1)^{2}}\right\} d t>0 .
$$

To prove (38) we divide the integrand into two parts, and estimate them separately. First, 


$$
\begin{aligned}
-\int_{-1}^{0} p(R ; t) d t & =\frac{1}{4}\left(R^{2}-1\right) \int_{0}^{1} \frac{1-t^{2}}{t^{1 / 2}(R+t)^{3 / 2}(R t+1)^{3 / 2}} d t \\
& <\frac{1}{4}\left(R^{2}-1\right) R^{-3 / 2} \int_{0}^{1} t^{-1 / 2}(R t+1)^{-3 / 2} d t \\
& =\frac{1}{2}\left(R^{2}-1\right) R^{-3 / 2}(R+1)^{-1 / 2} \\
& <\frac{1}{2}\left(R^{2}-1\right) R^{-2}<\frac{1}{2}\left(R^{2}-1\right) /\left(R^{2}-R+1\right)
\end{aligned}
$$

The absolute value of the remaining portion of the integral of (38) can be no larger than

$$
\frac{(R+1)^{2}}{4\left(R^{n}+1\right)} \int_{0}^{1} \frac{(1+t)^{2}\left(1+t^{n}\right)}{t^{1 / 2}(R+t)^{3 / 2}(R t+1)^{3 / 2}} d t .
$$

The integrand will be largest when $R=1$. Then, since $n \geqq 3$, (39) cannot exceed

$$
\frac{(R+1)^{2}}{4\left(R^{3}+1\right)} \int_{0}^{1} \frac{1+t^{3}}{t^{1 / 2}(1+t)} d t=\frac{13}{30} \frac{R+1}{R^{2}-R+1}
$$

6. Our assertion will now follow from the inequality

$$
\frac{1}{2}\left(R^{2}-1\right)+\frac{1}{3} \frac{3}{0}(R+1)<\frac{7}{6}\left(R^{2}-R+1\right), \quad R>1,
$$

which is easily verified. This proves (33).

The non-trivial character of (33) becomes clear from the remark that the inequality

$$
\sum_{m=0}^{n} c_{n-m}(R) \cos m \theta \geqq 0
$$

is not true for all real values of $\theta$ and $R \geqq 1$. Indeed [cf. Part I, (21)]

$$
\sum_{m=0}^{n}{ }^{\prime} c_{n-m}(1) \cos m \theta=\sum_{m=0}^{n}{ }^{\prime} \cos m \theta=\frac{1}{2} \sin n \theta \cot (\theta / 2) .
$$

This expression changes its sign at $\theta=\nu \pi / n, 1 \leqq \nu \leqq n$.

7. Since $C_{\nu n}>0,0 \leqq \nu \leqq n$, the equality sign in our main inequalities holds only for the special harmonic functions pointed out in the theorems of Part I. In order to discuss the conditions of equality in (3) we take into account the preliminary remarks of $\S 1$. Then we see that for $x=y=0, z=R$ the equality holds only if $(2 \pi)^{-1} \int_{-\pi}^{\pi} U(1, \theta, \phi) d \phi \equiv \pm \cos n \theta$. Since $|U| \leqq 1$ we conclude (cf. $[7$, p. 58]) that $U(1, \theta, \phi) \equiv \pm \cos n \theta$.

\section{Discussion of $c_{n}^{\prime}(1)$ AND $c_{n}^{\prime \prime}(1)$}

1. According to the results of the previous section, we have for $1 \leqq \nu \leqq n$ $C_{\nu n}(R)>0, R>1 ; C_{\nu n}(1)=0$; so $C_{\nu n}^{\prime}(1) \geqq 0$-that is, 


$$
\sum_{m=0}^{n}{ }^{\prime} c_{n-m}^{\prime}(1) \cos (m \nu \pi / n) \geqq 0,
$$

This is trivial for $\nu=0$ because $c_{n}^{\prime}(1)>0$. The constants $c_{n}^{\prime}(1)=\rho_{n}$ are identical with the constants introduced by Szegö [7, p. 60]. Inequalities (41) furnish the following theorem:

Let $u(x, y, z)$ be a harmonic polynomial of degree $n$ satisfying the condition $|u(x, y, z)| \leqq 1$ in $x^{2}+y^{2}+z^{2} \leqq 1$. Then on the unit sphere we have

$$
\left|\frac{\partial u}{\partial r}\right| \leqq c_{n}^{\prime}(1)=\rho_{n} .
$$

If the boundary values of $u$ on the unit sphere are of the form $\pm \cos n \gamma, \gamma$ being the spherical distance of the variable point from a fixed point of the unit sphere, then the equality occurs in (42).

This is slightly less than the main theorem of [7]. It is shown there that (42) is an equality only if $u \equiv \pm \cos n \gamma$.

The representation

$$
\rho_{n}=c_{n}^{\prime}(1)=\frac{1}{2} \pi n-\frac{1}{2}+\frac{(-1)^{n}}{2} \int_{0}^{1} \frac{(1-t) t^{n-1 / 2}}{(1+t)^{2}} d t
$$

is the analogue of (30). It follows from (30) by differentiation. Another way to obtain (43) is to calculate

$$
\left(\frac{\partial}{\partial R} F(R, z)\right)_{R=1}=\left(\frac{\partial}{\partial R} G(R, z)\right)_{R=1}=\sum_{n=1}^{\infty} \rho_{n} z^{n},
$$

and to apply to this function considerations similar to those in $\S \S 3,4$. This generating function was used in [7].

A direct proof of inequalities (41) is possible based on the representation (43).

2. Now let $\tau_{n}=\frac{1}{2} c_{n}^{\prime \prime}(1)$. We discuss the following inequalities, $n \geqq 2$,

$$
\begin{aligned}
\frac{1}{2} C_{\nu n}^{\prime \prime}(1)=\frac{1}{2} \sum_{m=0}^{n} c_{n-m}^{\prime \prime}(1) \cos (m \nu \pi / n)=\sum_{m=0}^{n} \tau_{n-m} \cos (m \nu \pi / n) & >0, \\
0 & \leqq \nu \leqq n .
\end{aligned}
$$

It was pointed out in $\S 1.3$ that $c_{n}^{\prime \prime}(1)>0$; so inequality (44) is trivial for $\nu=0$.

Let $1 \leqq \nu \leqq n$. It is advisable to calculate

$$
\left(\frac{\partial^{2}}{\partial R^{2}} F(R, z)\right)_{R=1}=\left(\frac{\partial^{2}}{\partial R^{2}} G(R, z)\right)_{R=1}+\sum_{n=1}^{\infty} n^{2} z^{n}=2 \sum_{n=2}^{\infty} \tau_{n} z^{n} .
$$

Introducing the abbreviation $(R-z)(1-R z)=K$ we obtain 


$$
\begin{aligned}
\frac{\partial}{\partial R} G(R, z)= & \left(1-z^{2}\right) z^{1 / 2}\left\{R K^{-3 / 2}\right. \\
& \left.-\frac{3}{4}\left(R^{2}-1\right) K^{-5 / 2}\left(1-2 R z+z^{2}\right)\right\} \arctan \left(\frac{z(R-z)}{1-R z}\right)^{1 / 2} \\
& +\left(1-z^{2}\right) z^{1 / 2 \frac{1}{2}\left(R^{2}-1\right) K^{-3 / 2 \frac{1}{2}} z^{1 / 2} K^{-1 / 2}}
\end{aligned}
$$

so $($ cf. $[7$, p. $60,(28)])$

$$
\left(\frac{\partial}{\partial R} G(R, z)\right)_{R=1}=\sum_{n=1}^{\infty} \rho_{n} z^{n}=z^{1 / 2}(1-z)^{-2}(1+z) \arctan \left(z^{1 / 2}\right) .
$$

Furthermore

$$
\begin{aligned}
\frac{\partial^{2}}{\partial R^{2}} G(R, z)= & \left(1-z^{2}\right) z^{1 / 2}\left\{K^{-3 / 2}-3 R K^{-5 / 2}\left(1-2 R z+z^{2}\right)\right\} \arctan \left(\frac{z(R-z)}{1-R z}\right)^{1 / 2} \\
& +\left(1-z^{2}\right) z^{1 / 2} R K^{-3 / 2} \frac{1}{2} z^{1 / 2} K^{-1 / 2} \\
& +\left(1-z^{2}\right) z^{1 / 2} R K^{-3 / 2 \frac{1}{2} z^{1 / 2} K^{-1 / 2}+\cdots}
\end{aligned}
$$

where the terms which are not written out vanish for $R=1$. This furnishes $\left(\frac{\partial^{2}}{\partial R^{2}} G(R, z)\right)_{R=1}=-2 z^{1 / 2}(1-z)^{-2}(1+z) \arctan \left(z^{1 / 2}\right)+z(1-z)^{-3}(1+z)$,

or

$$
\sum_{n=1}^{\infty}\left(2 \tau_{n}-n^{2}\right) z^{n}=-2 \sum_{n=1}^{\infty} \rho_{n} z^{n}+\sum_{n=1}^{\infty} n^{2} z^{n}
$$

so, on account of (43),

$$
\begin{aligned}
\tau_{n} & =\frac{1}{2} c_{n}^{\prime \prime}(1)=-\rho_{n}+n^{2} \\
& =n^{2}-\frac{1}{2} \pi n+\frac{1}{2}+\frac{(-1)^{n+1}}{2} \int_{0}^{1} \frac{(1-t) t^{n-1 / 2}}{(1+t)^{2}} d t .
\end{aligned}
$$

3. By use of formula (21) of Part I we find

$$
\sum_{m=0}^{n} m^{2} \cos m \theta=-\frac{1}{2}\left(\frac{d}{d \theta}\right)^{2}(\sin n \theta \cot (\theta / 2)) .
$$

Then for $1 \leqq \nu \leqq n$ we have

$$
\begin{aligned}
\sum_{m=0}^{n} \prime(n-m)^{2} & \cos (m \nu \pi / n) \\
= & \sum_{m=0}^{n}{ }^{\prime} m^{2} \cos (\nu \pi-m \nu \pi / n)=(-1)^{\nu} \sum_{m=0}^{n} m^{2} \cos (m \nu \pi / n) \\
= & (-1)^{\nu}\left(\frac{n \cos n \theta}{2 \sin ^{2}(\theta / 2)}\right)_{\theta=\nu \pi / n}=\frac{n}{2 \sin ^{2}(\nu \pi /(2 n))} .
\end{aligned}
$$


Also

$$
\sum_{m=0}^{n}(n-m) \cos (m \nu \pi / n)=\frac{1}{2}\left(\frac{\sin ^{2}(n \theta / 2)}{\sin ^{2}(\theta / 2)}\right)_{\theta=\nu \pi / n}=\frac{1-(-1)^{\nu}}{4 \sin ^{2}(\nu \pi /(2 n))},
$$

so from (45) [cf. Part I, (21), (20)] we obtain

$$
\begin{aligned}
\sum_{m=0}^{n}{ }^{\prime} \tau_{n-m} \cos m \theta & =\frac{n-\frac{1}{4} \pi\left(1-(-1)^{\nu}\right)}{2 \sin ^{2}(\theta / 2)} \\
& -\frac{1}{4} \int_{0}^{1} \frac{(1-t) t^{-1 / 2}}{(1+t)^{2}} \frac{1-t^{2}}{1+2 t \cos \theta+t^{2}}\left((-1)^{\nu}-(-t)^{n}\right) d t
\end{aligned}
$$

where $\theta=\nu \pi / n, 1 \leqq \nu \leqq n$.

Let $\nu$ be even. The first term as a function of $\cos \theta$ is increasing; the integral in the second term is decreasing. Therefore the total expression is greater than

$$
n / 2-\frac{1}{2} \int_{0}^{1} \frac{t^{-1 / 2}}{1+t} d t=n / 2-\pi / 4
$$

which is positive for $n \geqq 2$.

Let $\nu$ be $o d d$. Then, the integral in the second term of (46) is negative and $n-\pi / 2>0$, so the statement is clear. Thus, we have proved the following

Let $u(x, y, z)$ be a harmonic polynomial of degree $n$ satisfying the condition $|u(x, y, z)| \leqq 1$ in $x^{2}+y^{2}+z^{2} \leqq 1$. Then on the unit sphere

$$
\frac{1}{2}\left|\frac{\partial^{2} u}{\partial r^{2}}\right| \leqq \frac{1}{2} c_{n}^{\prime \prime}(1)=\tau_{n}
$$

with the sign "=" if and only if the boundary values of $u$ on the unit sphere are of the form $\pm \cos n \gamma, \gamma$ being the spherical distance of the variable point from a fixed point on the unit sphere.

7. Asymptotic Formula of $c_{n}(R)$ as $n \rightarrow \infty$

From (30) and $\left(30^{\prime}\right)$ we obtain for fixed $R>1$ and $n \rightarrow \infty$,

$$
\begin{aligned}
c_{n}(R) & =O\left(R^{n}\right)+\int_{1}^{R} p(R ; t)\left(R^{n}-t^{n}\right) d t \\
& =O\left(R^{n}\right)+\frac{\left(R^{2}-1\right)^{1 / 2}}{4 R^{1 / 2}} \int_{1}^{R} \frac{R^{n}-t^{n}}{(R-t)^{3 / 2}} d t+O(1) \int_{1}^{R} \frac{R^{n}-t^{n}}{(R-t)^{1 / 2}} d t \\
& =O\left(R^{n}\right)+\frac{\left(R^{2}-1\right)^{1 / 2}}{4 R^{1 / 2}} \int_{0}^{R} \frac{R^{n}-t^{n}}{(R-t)^{3 / 2}} d t \\
& =O\left(R^{n}\right)+\frac{\left(R^{2}-1\right)^{1 / 2}}{4 R} R^{n} \int_{0}^{1} \frac{1-t^{n}}{(1-t)^{3 / 2}} d t .
\end{aligned}
$$


But

$$
\begin{aligned}
\int_{0}^{1} \frac{1-t^{n}}{(1-t)^{3 / 2}} d t & =\sum_{m=0}^{n-1} \int_{0}^{1} \frac{t^{m}}{(1-t)^{1 / 2}} d t=2 \sum_{m=0}^{n-1} \frac{2 \cdot 4 \cdots 2 m}{3 \cdot 5 \cdots(2 m+1)} \\
& \simeq \sum_{m=1}^{n-1} \pi^{1 / 2} m^{-1 / 2} \simeq 2 \pi^{1 / 2} n^{1 / 2}, \quad n \rightarrow \infty,
\end{aligned}
$$

which furnishes (6).

Formula (6) also follows directly from (3).

\section{Appendix}

\section{Generalization}

A problem similar to that treated in Part II can be formulated for harmonic polynomials in any euclidean space. By using classical results on the representation of such harmonic polynomials in terms of ultraspherical polynomials $\left({ }^{10}\right)$, we are led to the following problem:

Let $P_{n}^{(\lambda)}(x)$ denote the ultraspherical polynomials, $\lambda>0$, and let $R$ be fixed, $R>1$. Considering all cosine polynomials

$$
u(\theta)=\sum_{m=0}^{n} \alpha_{m} P_{m}^{(\lambda)}(\cos \theta)
$$

of degree $n \geqq 1$, satisfying the condition $|u(\theta)| \leqq 1$ for real $\theta$, what is the maximum of $\left|\sum_{m=0}^{n} \alpha_{m} P_{m}^{(\lambda)}(1) R^{m}\right|$ ?

1. In case of harmonic polynomials of $p+1$ variables we have to take $\lambda=\frac{1}{2}(p-1)$. However the problem mentioned has sense for arbitrary $\lambda>0$. The two-dimensional case (Part I) corresponds to the limiting value $\lambda \rightarrow 0$, the three-dimensional case (Part II) to the value $\lambda=1 / 2$.

Again the maximum in question is attained for $u(\theta)= \pm \cos n \theta$ if and only if the following condition is satisfied. Let

$$
\cos n \theta=\sum_{m=0}^{n} h_{n m} P_{m}^{(\lambda)}(\cos \theta), \quad c_{n}^{(\lambda)}(R)=\sum_{m=0}^{n} h_{n m} P_{m}^{(\lambda)}(1) R^{m}
$$

then

$$
\sum_{m=0}^{n}{ }^{\prime} c_{n-m}^{(\lambda)}(R) \cos (m \nu \pi / n) \geqq 0, \quad 0 \leqq \nu \leqq n .
$$

2. The proof of this inequality for general $\lambda$ seems to be rather difficult.

(10) See, for instance, E. Heine, Handbuch der Kugelfunctionen, vol. 1 (2d edition, 1878); see in particular pp. 454 and 461. Regarding the notation of ultraspherical polynomials we follow Szegö's book quoted in Part II, \$2; see in particular p. 80. 
In the limiting case $\lambda \rightarrow 0 \mathrm{cf}$. formula (24) of Part I. In the case $\lambda=1 / 2$ we have inequality (33) of Part II.

Another simple case is $\lambda=1$. Since

$$
\cos n \theta=\frac{1}{2} \frac{\sin (n+1) \theta}{\sin \theta}-\frac{1}{2} \frac{\sin (n-1) \theta}{\sin \theta}, \quad n \geqq 1,
$$

we have

$$
c_{n}^{(1)}(R)=\frac{1}{2}(n+1) R^{n}-\frac{1}{2}(n-1) R^{n-2}, \quad n \geqq 1 ; \quad c_{0}^{(1)}(R)=1,
$$

so that

$$
\begin{aligned}
\sum_{m=0}^{n}{ }^{\prime} c_{n-m}^{(1)}(R) & \cos (m \nu \pi / n) \\
= & \frac{1}{2} \frac{d}{d R}\left\{\left(R-R^{-1}\right) \sum_{m=0}^{n}{ }^{\prime} R^{n-m} \cos (m \nu \pi / n)\right\} \\
& \quad-\frac{1}{4}(-1)^{\nu} \frac{d}{d R}\left(R-R^{-1}\right)+\frac{1}{2}(-1)^{\nu} \\
= & \frac{1}{2}\left(1+R^{-2}\right) \sum_{m=0}^{n}{ }^{\prime} R^{n-m} \cos (m \nu \pi / n) \\
& +\frac{1}{2}\left(1-R^{-2}\right) \sum_{m=0}^{n}(n-m) R^{n-m} \cos (m \nu \pi / n)+\frac{1}{4}(-1)^{\nu}\left(1-R^{-2}\right) .
\end{aligned}
$$

(6)

According to well known properties of Fejér's kernel, the second sum of the last expression is positive. The first sum is [cf. Part I, (20)]

$$
\frac{1}{2} \frac{\left(R^{2}-1\right)\left(R^{n}-(-1)^{\nu}\right)}{1-2 R \cos (\nu \pi / n)+R^{2}}
$$

This furnishes the positivity of (6) when $\nu$ is even. Let $\nu$ be odd; then we have only to show that

$$
\left(1+R^{-2}\right) \frac{\left(R^{2}-1\right)\left(R^{n}+1\right)}{1-2 R \cos (\nu \pi / n)+R^{2}}>1-R^{-2},
$$

which is clear.

Thus we established the following theorem:

Let $n \geqq 1$ and let

$$
u(\theta)=\sum_{m=0}^{n} \beta_{m} \frac{\sin (m+1) \theta}{\sin \theta}
$$


be an arbitrary cosine polynomial of degree $n$ satisfying the condition $|u(\theta)| \leqq 1$, $\theta$ real. Then for $R>1$

$$
\left|\sum_{m=0}^{n}(m+1) \beta_{m} R^{m}\right| \leqq \frac{1}{2}(n+1) R^{n}-\frac{1}{2}(n-1) R^{n-2}
$$

with the sign " =" if and only if $u(\theta)= \pm \cos n \theta$.

\section{A PROBLEM ON ELLIPSES}

1. Some time ago W. E. Sewell( $\left.{ }^{11}\right)$ dealt with the following extremum problem:

Let $E$ be an ellipse in the complex z-plane. If $f(z)$ is a polynomial of degree $n$ satisfying the condition $|f(z)| \geqq 1, z \in E$, what is the maximum of $\max \left|f^{\prime}(z)\right|$, $z \in E$ ?

Sewell obtains an upper bound for this maximum, which is, however, not the precise one. His bound is of order $n$ as $n \rightarrow \infty$, which is the precise order $\left({ }^{12}\right)$.

2. For sake of convenience we consider an ellipse $E_{R}$ with foci at -1 and +1 and with semi-axes $\frac{1}{2}\left(R \pm R^{-1}\right), R>1$. Let $\alpha=\frac{1}{2}\left(R+R^{-1}\right)$. We prove the following result of negative nature:

Let $n \geqq 5$. There exist polynomials $f(z)$ of degree $n$ satisfying the condition $|f(z)| \leqq 1, z \in E_{R}$, such that

$$
\left|f^{\prime}(\alpha)\right|>\frac{2 n}{R-R^{-1}} \frac{R^{n}-R^{-n}}{R^{n}+R^{-n}}=\frac{T_{n}^{\prime}(\alpha)}{T_{n}(\alpha)} .
$$

Here $T_{n}(z)$ is Tchebychef's polynomial; obviously $\left|T_{n}(z) / T_{n}(\alpha)\right| \leqq 1$ when $z \in E_{R}$. This shows that for $n \geqq 5$ the solution of the problem mentioned is not furnished by Tchebychef's polynomial. This is remarkable since Tchebychef's polynomial does furnish the maximum in question for all $n$ in the limiting case $R \rightarrow 1$, that is, in the case of the segment $(-1,+1)$.

3. Let $2 z=R e^{i \phi}+R^{-1} e^{-i \phi}$. If $\phi$ runs from 0 to $2 \pi, z$ describes $E_{R} ; \phi=0$ furnishes $z=\alpha$. We have with certain complex coefficients $c_{m}$

$$
\begin{aligned}
f(z) & =\sum_{m=0}^{n} c_{m}\left(R^{m} e^{i m \phi}+R^{-m} e^{-i m \phi}\right) \\
& =\sum_{m=0}^{n} c_{m}\left(R^{m}+R^{-m}\right) \cos m \phi+i \sum_{m=0}^{n} c_{m}\left(R^{m}-R^{-m}\right) \sin m \phi, \\
f^{\prime}(\alpha) & =\sum_{m=0}^{n} 2 m c_{m} \frac{R^{m}-R^{-m}}{R-R^{-1}} .
\end{aligned}
$$

(11) W. E. Sewell, On the polynomial derivative constant for an ellipse, American Mathematical Monthly, vol. 44 (1937), pp. 577-578.

(12) G. Szegö, Über einen Satz yon A. Markoff, Mathematische Zeitschrift, vol. 23 (1925), pp. 45-61; see in particular p. 53. 
Substituting in the interpolation formula of Part I, §2:

$$
\lambda_{m}=\frac{2 m}{R-R^{-1}} \frac{R^{m}-R^{-m}}{R^{m}+R^{-m}}, \quad \mu_{m}=0, \quad 0 \leqq m \leqq n,
$$

we obtain

$$
\begin{aligned}
f^{\prime}(\alpha) & =\frac{1}{2 n} \sum_{\nu=0}^{2 n-1}(-1)^{\nu} G_{\nu} f\left(z_{\nu}\right), \quad z_{\nu}=\frac{1}{2}\left(R e^{i \nu \pi / n}+R^{-1} e^{-i \nu \pi / n}\right), \\
G_{\nu} & =\frac{4}{R-R^{-1}} \sum_{m=0}^{n}(n-m) \frac{R^{n-m}-R^{-(n-m)}}{R^{n-m}+R^{-(n-m)}} \cos (m \nu \pi / n) .
\end{aligned}
$$

4. Our next purpose is to show that for $n \geqq 5$, for some $\nu=\nu(n, R)$ and for sufficiently large values of $R$, the inequality $G_{\nu}<0$ holds. Indeed as $R \rightarrow \infty$, $1 \leqq \nu \leqq n$,

$$
\frac{1}{4}\left(R-R^{-1}\right) G_{\nu}=\sum_{m=0}^{n}(n-m) \cos (m \nu \pi / n)-2 R^{-2} \cos ((n-1) \nu \pi / n)+O\left(R^{-4}\right)
$$

$$
=\frac{1}{2} \frac{1-(-1)^{\nu}}{1-\cos (\nu \pi / n)}-2 R^{-2} \cdot(-1)^{\nu} \cos (\nu \pi / n)+O\left(R^{-4}\right) .
$$

Let $\nu$ be even and $0<\nu<n / 2$; then the last expression becomes negative if $R$ is sufficiently large. The condition regarding $\nu$ implies $n / 2>2, n \geqq 5$, and indeed for $n \geqq 5$ values $\nu=\nu(n)$ of the kind mentioned always exist.

5. Finally let $n \geqq 5, G_{\nu(n)}<0$ ( $R$ sufficiently large) and

$$
f(z)=T_{n}(z) / T_{n}(\alpha)-\eta h(z)
$$

where $\eta>0$ and $h(z)$ is a polynomial of degree $n$ satisfying the following conditions:

(a) that $h(z)$ has real coefficients;

(b) that $(-1)^{\nu} \Re h\left(z_{\nu}\right)>0,0 \leqq \nu \leqq 2 n-1$;

(c) that

$$
\begin{aligned}
h^{\prime}(\alpha) & =\frac{1}{2 n} \sum_{\nu=0}^{2 n-1}(-1)^{\nu} G_{\nu} h\left(z_{\nu}\right) \\
& =\frac{1}{2 n} \Re\left\{G_{0} h\left(z_{0}\right)+2 \sum_{\nu=1}^{n-1}(-1)^{\nu} G_{\nu} h\left(z_{\nu}\right)+(-1)^{n} G_{n} h\left(z_{n}\right)\right\}<0 .
\end{aligned}
$$

In (15) we have taken into account that $G_{n+\mu}=G_{n-\mu}, z_{n+\mu}=\bar{z}_{n-\mu}$, which follows from (12).

Such a polynomial $h(z)$ can easily be constructed. We write if $z \in E_{R}$

$$
h(z)=\sum_{m=0}^{n} h_{m}\left(R^{m} e^{i m \phi}+R^{-m} e^{-i m \phi}\right) ;
$$


the coefficients $h_{m}$ being real,

$$
\Re h\left(z_{\nu}\right)=\sum_{m=0}^{n} h_{m}\left(R^{m}+R^{-m}\right) \cos (m \nu \pi / n) .
$$

Now we determine the real coefficients $h_{m}$ such that $\Re h\left(z_{\nu}\right)$ assumes preassigned values for $0 \leqq \nu \leqq n$, in particular such that (b) is satisfied for $0 \leqq \nu \leqq n$; then the same condition (b) is satisfied for $0 \leqq \nu \leqq 2 n-1$ since $z_{n+\mu}=\bar{z}_{n-\mu}$. Finally (c) follows if the further condition is imposed that $(-1)^{\nu} \Re h\left(z_{\nu}\right)$ should be so large for $\nu=\nu(n)$ as to furnish the dominant term in $h^{\prime}(\alpha)$.

Since $\max \left|T_{n}(z)\right|, z \in E_{R}$, is attained only for $z=z_{\nu}, 0 \leqq \nu \leqq 2 n-1$, we obtain by a familiar argument, $|f(z)| \leqq 1, z \in E_{R}$, provided $\eta$ is sufficiently small. From

$$
f^{\prime}(\alpha)=T_{n}^{\prime}(\alpha) / T_{n}(\alpha)-\eta h^{\prime}(\alpha)
$$

we conclude that $\left|f^{\prime}(\alpha)\right|>T_{n}^{\prime}(\alpha) / T_{n}(\alpha)$ provided $\eta$ is sufficiently small.

This establishes the result stated.

\section{Bibliography}

1. S. Bernstein, Sur un théorème de M. Szegö, Prace Matematyczno-Fizyczne, vol. 44 (1937), pp. 9-14.

2. M. Riesz, Eine trigonometrische Interpolationsformel und einige Ungleichungen für Polynome, Jahresbericht der deutschen Mathematiker-Vereinigung, vol. 23 (1915), pp. 354-368.

3. G. Szegö, Über trigonometrische und harmonische Polynome, Mathematische Annalen, vol. 79 (1919), pp. 323-339.

4. — Über einen Satz des Herrn S. Bernstein, Schriften der Königsberger Gelehrten Gesellschaft, 1928, pp. 59-70.

5. - Über trigonometrische Interpolation, Schriften der Königsberger Gelehrten Gesellschaft, 1928, pp. 71-80.

6. - Solution to Problem 3705 (proposed by Raphael Robinson), American Mathematical Monthly, vol. 43 (1936), pp. 246-259.

7. - On the gradient of solid harmonic polynomials, these Transactions, vol. 47 (1940), pp. 51-65.

StANFoRd UNiversity,

Stanford University, Calif. 